\title{
Transsynaptic Signaling by Postsynaptic Synapse-Associated Protein 97
}

\author{
Maria Paz Regalado, Ryan T. Terry-Lorenzo, Clarissa L. Waites, Craig C. Garner, and Robert C. Malenka \\ Department of Psychiatry and Behavioral Sciences, Nancy Pritzker Laboratory, Stanford University, Palo Alto, California 94304-5485
}

The molecular mechanisms by which postsynaptic modifications lead to precisely coordinated changes in presynaptic structure and function are primarily unknown. To address this issue, we examined the presynaptic consequences of postsynaptic expression of members of the membrane-associated guanylate kinase family of synaptic scaffolding proteins. Postsynaptic expression of synapse-associated protein 97 (SAP97) increased presynaptic protein content and active zone size to a greater extent than comparable amounts of postsynaptic PSD-95 (postsynaptic density-95) or SAP102. In addition, postsynaptic expression of SAP97 enhanced presynaptic function, as measured by increased FM4-64 dye uptake. The structural presynaptic effects of postsynaptic SAP97 required ligand binding through two of its PDZ (PSD-95/Discs large/zona occludens-1) domains as well as intact N-terminal and guanylate kinase domains. Expression of SAP97 recruited a complex of additional postsynaptic proteins to synapses including glutamate receptor 1, Shank1a, SPAR (spineassociated RapGAP), and proSAP2. Furthermore, inhibition of several different transsynaptic signaling proteins including cadherins, integrins, and EphB receptor/ephrinB significantly reduced the presynaptic growth caused by postsynaptic SAP97. These results suggest that SAP97 may play a central role in the coordinated growth of synapses during development and plasticity by recruiting a complex of postsynaptic proteins that enhances presynaptic terminal growth and function via multiple transsynaptic molecular interactions.

Key words: synapse; active zone; retrograde signal; scaffolding proteins; cell adhesion; PSD-95

\section{Introduction}

Excitatory synapses in the mammalian brain are formed by presynaptic and postsynaptic specializations designed for rapid and efficient information transfer between neurons. A remarkable feature of such synapses is the precise alignment between the active zone (AZ), the presynaptic specialization at which vesicles dock and fuse with the plasma membrane, and the postsynaptic density (PSD), the specialization containing glutamate receptors (GluRs) and associated signaling machinery. Indeed, the sizes of AZs and PSDs are precisely matched at individual synapses (Lisman and Harris, 1993). The molecular mechanisms for this precise correspondence between presynaptic and postsynaptic specializations are unknown but likely involve site-specific recruitment of macromolecular protein complexes (Garner et al., 2002; Li and Sheng, 2003; Scheiffele, 2003). These mechanisms are important both for synaptogenesis and the structural changes at synapses that accompany synaptic plasticity (Engert and Bonhoeffer, 1999; Yuste and Bonhoeffer, 2001; Matsuzaki et al., 2004; Nagerl et al., 2004; Zhou et al., 2004).

How activity-dependent postsynaptic modifications lead to long-lasting structural changes in synapse size is unknown, but

Received Nov. 1, 2005; revised Jan. 13, 2006; accepted Jan. 14, 2006.

This work was supported by National Institutes of Health Grants MH063394 (R.C.M.) and HD38760 (C.C.G.) and by Ruth L. Kirchstein National Research Service Awards (R.T.T.-L. and C.L.W.). M.P.R. was the recipient of a postdoctoral fellowship from the Spanish Ministry of Education, Culture and Sport. We thank T. Lizama, E. Saura, J. Fisher, S. Wu, and S. Y. Lee for excellent technical assistance.

Correspondence should be addressed to Robert C. Malenka, Department of Psychiatry and Behavioral Sciences, Stanford Medical Center, 1201 Welch Road, Palo Alto, CA 94304-5485. E-mail: malenka@stanford.edu. DOI:10.1523/JNEUROSCI.5247-05.2006

Copyright $\odot 2006$ Society for Neuroscience $\quad$ 0270-6474/06/262343-15\$15.00/0 the process must involve retrograde signaling from postsynaptic to presynaptic sides of the synapse. In this context, a postsynaptic protein that has received great attention is synapse-associated protein 90 (SAP90)/PSD-95, a member of the family of membrane-associated guanylate kinase (GK) homologs (MAGUKs). When overexpressed in cultured neurons, PSD-95 causes increases in both postsynaptic AMPA receptor (AMPAR) number and presynaptic function (El-Husseini et al., 2000). Although the postsynaptic effects of PSD-95 are known to involve interactions with stargazin/transmembrane AMPA receptor regulatory proteins (Chen et al., 2000; Schnell et al., 2002), nothing is known about the mechanisms by which PSD-95 influences presynaptic function or whether other MAGUKs exert similar effects.

In vertebrates, three additional members of the SAP90/ PSD-95 family are expressed including SAP97/hDLG (human homolog of Drosophila Discs large), PSD-93/Chapsyn-110, and SAP102/NE-dlg. Each shares a similar domain structure, yet little is known about their synaptic functions (Montgomery et al., 2004). Arguably, the next best-studied MAGUK is SAP97, which can influence synaptic AMPARs when overexpressed postsynaptically (Rumbaugh et al., 2003; Nakagawa et al., 2004) and perhaps also affect presynaptic function (Rumbaugh et al., 2003). In the present study, we investigated how postsynaptic expression of SAP90/PSD-95 family members alters presynaptic structure. We find that compared with SAP90/PSD-95 and SAP102, postsynaptic SAP97 has the most potent presynaptic effect, dramatically increasing levels of the active zone protein Bassoon and the vesicle proteins synapsin and synaptophysin. Recruitment of these presynaptic proteins dramatically affects presynaptic function, as 
evidenced by a marked increase in $N$-(3-triethylammoniumpropyl)-4-(6-(4-(diethylamino)phenyl) hexatrienyl) pyridinium dibromide (FM4-64) dye uptake. Structure/function studies of SAP97 indicate that this transsynaptic effect requires functional PDZ (PSD-95/Dlg/zona occludens-1) domains as well as intact S97N and GK domains. Increasing postsynaptic SAP97 also cause a selective increase of several binding partners including the AMPAR subunit GluR1 and the scaffold proteins ProSAP2, Shank1a, and SPAR/SPAL (spine-associated RapGAP/SPA-1-like protein). Furthermore, our results suggest that the transsynaptic effects of postsynaptic SAP97 require multiple cell adhesion and signaling molecules including cadherins, integrins, and EphB receptor/ ephrinB. These results suggest that SAP97 plays a central role in coordinating the growth and functional organization of the presynaptic and postsynaptic components of synapses during both development and synaptic plasticity.

\section{Materials and Methods}

Transfection and treatment of hippocampal cell cultures. Medium density hippocampal cultures were prepared from postnatal day 0 Sprague Dawley rat pups as described previously (Yu and Malenka, 2003). Neurons were plated in culture medium consisting of Neurobasal medium (Invitrogen, Carlsbad, CA), $2 \times$ B-27 (Invitrogen), and 2 mм Glutamax-I (Invitrogen) at a density of $37,000-56,000$ cells $/ \mathrm{cm}^{2}$ on $12 \mathrm{~mm}$ coverslips coated with poly-D-lysine (Sigma, St. Louis, MO). Calcium phosphate transfections were performed at $7 \mathrm{~d}$ in vitro (div) using 1.5-3 $\mu \mathrm{g}$ of DNA per 24-well plate and fixed for analysis $72 \mathrm{~h}$ later. For all treatments, pharmacological reagents were added to the conditioned cultured media after washing out the calcium phosphate precipitate (40-90 min after transfection) and were replenished every day until processed for immunocytochemistry. Pharmacological reagents were used at the following concentrations: 5-15 $\mu \mathrm{g} / \mathrm{ml}$ Ephrin-B1/Fc (R \& D Systems, Minneapolis, $\mathrm{MN}), 15 \mu \mathrm{g} / \mathrm{ml}$ EphrinA5/Fc (R \& D Systems), 1:50 to 1:100 dilutions of monoclonal anti-N-cadherin antibody (clone GC-4; Sigma), $1 \mathrm{~mm}$ GRGDSP peptide (Calbiochem, La Jolla, CA), $50 \mu \mathrm{g} / \mathrm{ml} \beta$-neurexin/Fc, and $50 \mu \mathrm{g} / \mathrm{ml}$ human IgG (gift from T. Südhof, University of Texas Southwestern Medical Center, Dallas, TX).

Mammalian expression constructs. Plasmids encoding green fluorescent protein (GFP)-tagged SAP97 constructs, including all deletion mutants, have been described previously (Wu et al., 1998). All GFP-SAP97 constructs in this study contained the I3 insert in the loop between Src homology 3 (SH3) and GK domains and lack the I1A insert in the Nterminus domain (Lue et al., 1994; Muller et al., 1995; McLaughlin et al., 2002). Single [F203H (PDZ1), F298H (PDZ2), and F470H (PDZ3)] point mutants in the PDZ domains of SAP97 were made with the QuickChange site-directed mutagenesis kit (Stratagene, La Jolla, CA) using pBluescript SKII(-) encoding full-length SAP97 as a template. Then, using internal restriction sites, different DNA fragments encoding SAP97 in the pEGFP-C1-SAP97 plasmid were exchanged to generate single, double, and triple PDZ point mutants. All mutations were confirmed by sequencing. The PSD-95-GFP-tagged construct was produced by subcloning the cDNA encoding a myc-tagged version of PSD-95 (gift from D. Clapham, Howard Hughes Medical Institute and Children's Hospital, Boston, MA) into pEGFP-N1 (Clontech, Mountain View, CA). The SAP102-GFP-tagged construct was produced by subcloning full-length SAP102 into pEGFP-N3 (Clontech).

Immunostaining. Transfected cells were examined $3 \mathrm{~d}$ after transfection (10 div). Neurons were fixed for immunocytochemistry in $3.7 \%$ formaldehyde at room temperature for 20 min except when staining for PSD-95, Shankla, SPAR/SPAL, and Homer1b,c which required $100 \%$ methanol at $-20^{\circ} \mathrm{C}$ for $15 \mathrm{~min}$. Neurons were permeabilized for $5 \mathrm{~min}$ at room temperature with $0.25 \%$ Triton-X 100 except for the surface GluR1 staining experiments. After three washes in PBS/0.1 M glycine, neurons were blocked in $3 \%$ normal goat serum $/ 3 \%$ normal donkey serum/PBS or a blocking solution containing $0.2 \%$ gelatin, $2 \%$ glycine, $2 \%$ BSA, 50 $\mathrm{mm} \mathrm{NH}_{4} \mathrm{Cl}$ in PBS, $\mathrm{pH} 7.4-7.5$, for $1 \mathrm{~h}$ at room temperature. Primary antibodies were added in blocking buffer and incubated for $1 \mathrm{~h}$ at room temperature or overnight at $4^{\circ} \mathrm{C}$.

The following primary antibodies and dilutions were used: rabbit antimicrotubule-associated protein 2 (anti-MAP2; 1:1500; Chemicon, Temecula, CA), mouse anti-MAP2 (1:800; Sigma), mouse anti-PSD-95 (1: 100; Sigma), rat anti-Homer $1 \mathrm{~b}, \mathrm{c}$ (1:100; Chemicon), rabbit antisynaptohysin (1:2000; Zymed, San Francisco, CA), mouse monoclonal anti-synapsin (1:2000; Chemicon), rabbit anti-SPAL (1:100; gift from T. Akiyama, Institute for Molecular and Cellular Biosciences, University of Tokyo, Tokyo, Japan), rabbit anti-Shank-1A (1:100; gift from M. Sheng, Massachusetts Institute of Technology, Cambridge, MA), rabbit antibody directed at the N terminus of GluR1 (1:1000; gift from R. Huganir, Johns Hopkins University School of Medicine, Baltimore, MD), rabbit anti-ProSAP2/Shank3 antibodies (1:100; gift from Tobias Boecher, Magdeburg, Germany). Several Bassoon antibodies were used: a rabbit Bassoon antisera (1:100) that was described previously (tom Dieck et al. 1998) and mouse monoclonal anti-Bassoon (1:500 to1:2000; StressGen, Victoria, British Columbia, Canada). After three washes in PBS, neurons were incubated at least $30 \mathrm{~min}$ in blocking solution and then incubated with secondary antibodies in blocking solution for $1 \mathrm{~h}$ at room temperature. Secondary antibodies included Alexa Fluor 568 goat anti-mouse IgG (1:800), Alexa Fluor 568 goat anti-rabbit IgG (1:800; Invitrogen), cyanine 5 (Cy5)-conjugated AffinityPure donkey anti-mouse (1:500), and Cy5-conjugated AffinityPure donkey anti-rabbit (1:500; Jackson ImmunoResearch, West Grove, PA). Cy5-conjugated secondary antibodies were used for all endogenous protein staining except for surface GluR1 staining, where Alexa 568 was used.

Imaging and data analysis. Images were acquired on a Zeiss (Oberkochen, Germany) LSM 510 laser scanning confocal microscope using a $40 \times$ oil immersion Neofluor objective [numerical aperture (NA), 1.3] for Figures 1 and 2 or $63 \times$ oil immersion Plan Achromat objective (NA, 1.4) for Figures 3 and 5-8. Each image was a $z$ series projection of five to eight sections each averaged twice and taken at $0.58 \mu \mathrm{m}$ depth intervals, except for Figures 1 and 2 analyses, in which each image was a single image averaged twice. Because of the variability of GFP intensity in transfected cells, we adjusted the detection gain in the GFP channel so the image was not saturated. Transfected neurons were chosen randomly for quantification from two to six coverslips from at least three independent experiments for each construct or at least two for each treatment. The number of neurons or puncta used for quantification is indicated in the figures. Images were analyzed with MetaMorph image analysis software (Universal Imaging Corporation, West Chester, PA) and processed using Adobe Photoshop (Adobe Systems, San Jose, CA) software for presentation. For the puncta intensity analysis, images were separated into individual channels by MetaMorph imaging software, thresholded for the channel under analysis, and analyzed using the Integrated Morphometry Analysis tool. Proximal dendrites were detected by MAP2 staining, except for surface GluR1 staining images, and were selected randomly for transfected and untransfected neurons on the same image. Individual puncta intensity measurements were averaged per dendrite, and the ratio between values in transfected cells with those in untransfected cells was calculated. Ratio means from several neurons were averaged to calculate population mean (presented as percentage of untransfected cells \pm SEM). For all figures (except Figs. 1 and 2), puncta of endogenous proteins colocalizing with the GFP-tagged overexpressed proteins were analyzed separately from puncta that did not colocalize. For the data in Figures 1 and 2, all puncta along the dendrites were included in the analysis, and two dendrites from each transfected and untransfected neurons were analyzed and the average calculated. For correlation and ratio analyses, only images acquired on the same day with the same acquisition parameters for both GPF and Bassoon channels were used, images were thresholded for both channels, and the puncta intensity was analyzed in the overlay image using the Region Measurements tool in MetaMorph Software. Statistical significance was determined using two-tailed Student's $t$ test or one-way ANOVA test and a Tamhane post test, as indicated in figure legends.

FM4-64 loading and fluorescence imaging. Transfected cells were examined $3 \mathrm{~d}$ after transfection (10 div). Coverslips containing SAP97transfected neurons were placed in a custom-built imaging chamber (S. J. 
Smith, Stanford University) and perfused with Tyrode's saline solution (in mM: $119 \mathrm{NaCl}, 2.5 \mathrm{KCl}, 2 \mathrm{CaCl}_{2}, 2 \mathrm{MgCl}_{2}, 30$ glucose, $25 \mathrm{HEPES}, \mathrm{pH}$ 7.4) containing $50 \mu \mathrm{M}$ APV and $10 \mu \mathrm{M}$ CNQX (Tocris Cookson, Ballwin, MO). The styryl dye FM4-64 (Invitrogen) was then added at a concentration of $15 \mu \mathrm{M}$. Synaptic vesicles were loaded with FM4-64 using $10 \mathrm{~Hz}$ electrical stimulation for $30 \mathrm{~s}$, followed by a $30 \mathrm{~s}$ pause to allow for maximal dye uptake. Cells were then washed with normal Tyrode's solution for several minutes to decrease background staining of membranes with FM4-64. Imaging was done with a scanning laser confocal microscope designed by S. J. Smith (Stanford University), using a Zeiss $63 \times 1.4$ NA Plan Achromat objective. Microscope control software was written by N. E. Ziv (Rappaport Institute, Haifa, Israel). Two or three $z$-sections taken at $1.0 \mu \mathrm{m}$ depth intervals were obtained for each image. The intensities of fluorescence puncta were analyzed using MetaMorph image analysis software (Universal Imaging Corporation) and processed using Adobe Photoshop software for presentation. Images for both GFP and FM4-64 channels were thresholded and combined by MetaMorph imaging software. A square region of $350 \times 350$ pixels was traced around the GFP-SAP97-transfected cell, and FM4-64 puncta in that region were identified automatically with the Create Regions Around Objects tool. The individual FM4-64 puncta intensity was analyzed using the Region Measurements tool in MetaMorph Software. For each experiment, the FM4-64 fluorescence of all GFP-SAP97-expressing puncta was normalized by dividing by the average FM4-64 fluorescence of the non-GFPSAP97-expressing control puncta. Normalized FM4-64 puncta intensity values from seven images (three different culture preparations) were averaged to calculate population mean \pm SEM. Statistical significance was determined using two-tailed Student's $t$ test.

Expression and purification of glutathione S-transferase fusion proteins. The pGEX-2TN plasmid (modified from pGEX-2T by changing the multiple cloning site) encoding the C-terminal region of NR2B, containing amino acid residues $1474-1482$, or the C-terminal region of cysteinerich interactor of PDZ3 (CRIPT), containing amino acid residues 93-101 was transformed into BL21(DE3)pLysS Escherichia coli (Invitrogen), and the bacteria were grown in LB media to $A_{600}=0.6$. Protein expression was induced by the addition of $0.1 \mathrm{~mm}$ isopropyl-1-thio- $\beta$-Dgalactopyranoside to the media and continued growth for $3 \mathrm{~h}$ at $37^{\circ} \mathrm{C}$. Cells were lysed by sonication, and glutathione $S$-transferase (GST) fusion proteins were affinity-purified using glutathione-agarose beads (Sigma) according to the manufacturer's instructions. Protein concentration was determined with a Bradford assay (Bio-Rad, Hercules, CA).

Cell lysate preparation. Human embryonic kidney 293 (HEK293) cells were maintained in DMEM supplemented with $10 \%$ fetal bovine serum and penicillin-streptomycin at $37^{\circ} \mathrm{C}$ in $5 \% \mathrm{CO}_{2}$. Cells grown to $50-70 \%$ confluence in $10 \mathrm{~cm}$ plates were transiently transfected with $6 \mu \mathrm{g}$ of each DNA encoding GFP, GFP-SAP97, GFP-SAP97PDZ1\&2FH, GFPSAP97PDZ1\&3FH, GFP-SAP97PDZ2\&3FH, or GFP-SAP97PDZ123FH using LipofectAMINE (Invitrogen), according to the manufacturer's instructions. After $18-24 \mathrm{~h}$, cells were washed with PBS and lysed in $2 \mathrm{ml}$ of lysis buffer (50 mu Tris, pH 7.5, 1 mм EDTA, $150 \mathrm{~mm} \mathrm{NaCl}, 1 \%$ Triton $\mathrm{X}-100$, and $0.1 \% \beta$-mercaptoethanol) containing complete protease inhibitor mixture (Roche Products, Welwyn Garden City, UK) for 20 min on ice. Lysates were removed and cleared by centrifugation at $20,000 \times g$ for $10 \mathrm{~min}$ at $4^{\circ} \mathrm{C}$ before performing an in vitro pull-down assay.

Pull-down assays. For sedimentation or pull-down assays with GST fusion proteins, glutathione-Sepharose (bed volume, $30 \mu \mathrm{l}$ ) was equilibrated with TBS (10 mm Tris- $\mathrm{HCl}, \mathrm{pH} 7.5$, containing $150 \mathrm{~mm} \mathrm{NaCl}$ ). GST proteins were added ( $5 \mu \mathrm{g})$, and the volume was adjusted to $300 \mu \mathrm{l}$ with TBS before incubation for $1 \mathrm{~h}$ at $4^{\circ} \mathrm{C}$. The beads were washed once with TBS, and HEK293 cell lysates expressing GFP, GFP-SAP97, or the different GFP-SAP97 PDZ mutants ( $200 \mu \mathrm{l}$ of $2 \mathrm{ml}$ total volume) were added, and the mixture was incubated for $1 \mathrm{~h}$ at $4^{\circ} \mathrm{C}$. The beads were washed four times with NETN-250 (10 mm Tris-HCl, pH 7.5, $1 \mathrm{~mm}$ EDTA, $250 \mathrm{~mm} \mathrm{NaCl}$, and $0.5 \%$ Nonidet P-40). Bound proteins were eluted using $40 \mu \mathrm{l}$ of SDS sample buffer, separated by SDS-PAGE on $10 \%$ (w/v) acrylamide gels, and subjected to immunoblot analysis with rabbit polyclonal anti-GFP antibody (Invitrogen). Coomassie blue staining verified the loading of GST and GST fusion proteins on beads.

\section{Results \\ Postsynaptic SAP97 enhances the accumulation of presynaptic proteins}

In a previous study, increasing the expression of SAP97 postsynaptically not only increased the amount of GluR1 at synapses but also induced a significant increase in the frequency of miniature EPSCs (mEPSCs) (Rumbaugh et al., 2003). An unresolved issue of that study was whether the increase in MEPSC frequency was caused by an increase in the levels of AMPARs at individual synapses, an increase in synapse number, or an increase in the size/ efficacy of individual presynaptic boutons. Thus, in the present study, we sought to define the mechanisms whereby the overexpression of postsynaptic SAP97 influenced presynaptic terminal growth/development. This was initially accomplished by transiently expressing a GFP-tagged version of SAP97 containing the I3 insert (Lue et al., 1994; McLaughlin et al., 2002) in cultured hippocampal neurons. When cultures were examined $3 \mathrm{~d}$ after transfection, GFP-SAP97 displayed a punctate pattern of expression in the dendrites of transfected cells. On average $>80 \%$ of these puncta were found to colocalize with clusters of presynaptic vesicle and active zone proteins (Fig. 1). To quantify the effect of GFP-SAP97 on presynaptic terminal growth or number, cultures were fixed and stained with antibodies against synaptophysin, a synaptic vesicle protein, synapsin, a protein thought to link synaptic vesicles to the actin cytoskeleton, and Bassoon, a core component of the active zone (Volknandt, 1995; Sudhof, 2004). We measured the average puncta intensity and number of puncta per unit length of dendrites from single confocal images, using MAP2 staining to outline the dendritic processes (Fig. $1 A-F$ ). When compared with neighboring untransfected cells in the same microscopic field, neurons expressing GFP-SAP97 showed an increase in the intensity and number of synaptophysin puncta $(167 \pm 10162 \pm 10 \%$, respectively; $n=52$ cells) (Fig. $1 A, G, H)$, an increase in the intensity and number of synapsin puncta $(135 \pm 5$ and $146 \pm 10 \%$, respectively; $n=76$ ) (Fig. $1 C, G, H)$, and an increase in the intensity and number of Bassoon puncta $(154 \pm 7$ and $188 \pm 21 \%$, respectively; $n=61$ ) (Fig. $1 E, G, H)$. In contrast, GFP-expressing neurons exhibited no significant change in average puncta intensity and number of puncta per unit length for any of these presynaptic proteins (Fig. $1 B, D, F-$ $H)$. These results demonstrate that postsynaptic overexpression of SAP97 in hippocampal neurons not only increases the number of synapses formed on these cells but also promotes a robust accumulation of three different classes of presynaptic proteins. Although the measured intensity of the epi-fluorescence does not necessarily reflect presynaptic bouton and active zone size in an absolute quantitative sense, these measurements are consistent with a relative increase in the size of both of these compartments. As such, these data suggest a role for postsynaptic SAP97 in regulating the assembly and growth of the presynaptic apparatus.

\section{SAP97 has greater presynaptic effects than SAP90/PSD-95 or SAP102}

Because all the members of the SAP90/PSD-95 family of proteins are highly homologous and SAP90/PSD-95 can also influence presynaptic development (El-Husseini et al., 2000), we next examined how the presynaptic effects of postsynaptic SAP97 compared with those of other family members. To address this question, we compared, under identical experimental conditions, the effects of expressing SAP102-GFP, PSD-95-GFP, and GFPSAP97 on average Bassoon puncta intensity (Fig. 2). We chose Bassoon as the presynaptic marker of choice, because as a core component of the active zone (tom Dieck et al., 1998; Sudhof, 


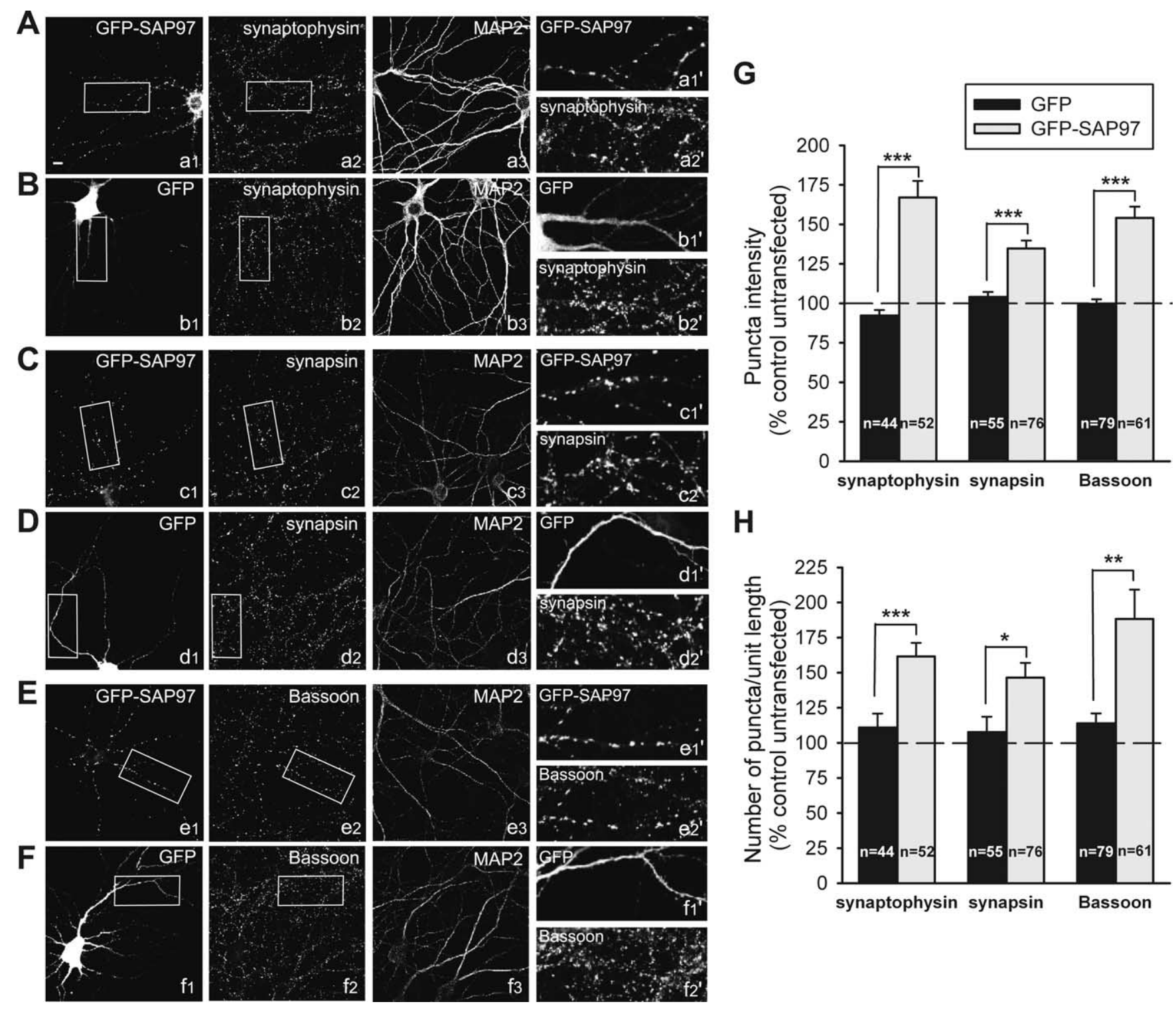

Figure 1. Postsynaptic expression of SAP97 enhances expression of presynaptic proteins. $\boldsymbol{A}-\boldsymbol{F}$, Representative images of hippocampal neurons transfected with GFP-SAP97 (a1, $\boldsymbol{c 1}, \boldsymbol{e} 1)$ or GFP $(\boldsymbol{b} \mathbf{1}, \mathbf{d} \mathbf{1}, \mathbf{f 1})$ and double stained for synaptophysin $(\boldsymbol{a} \mathbf{2}, \boldsymbol{b 2})$, synapsin $(\mathbf{c} \mathbf{2}, \mathbf{2})$, or Bassoon $(\boldsymbol{e} 2, \mathbf{f 2})$ and MAP2 $(\boldsymbol{a} \mathbf{3}-\mathbf{f 3})$ to visualize the dendrites. Individual channels are shown in grayscale for better resolution. Enlargements of the boxed regions are shown in the respective right panels $\left(\boldsymbol{a 1 ^ { \prime }}-\mathbf{f 2 ^ { \prime }}\right)$. Scale bar: (in $\left.\boldsymbol{a 1}\right) \mathbf{1 0} \mu \mathrm{m}$. $\mathbf{G}$, Quantitative analysis of changes in the average puncta intensity of the indicated presynaptic protein induced by overexpression of GFP-SAP97 (gray bars) or GFP (black bars). Bars show mean \pm SEM expressed as percentage of average puncta intensity in neighboring untransfected neurons in the same microscopic field ( $n=$ number of cells). ${ }^{* * *} p<0.0005$ (two-tailed $t$ test). $\boldsymbol{H}$, Quantitative analysis of changes in the number of puncta per unit length of the indicated presynaptic protein in the same neurons as in $\mathbf{G} .{ }^{* * *} p<0.0005,{ }^{* *} p<0.005,{ }^{*} p<0.05$; two-tailed $t$ test.

2004), its amount is likely to reflect the relative size of active zones. Surprisingly, although expression of PSD-95-GFP significantly increased average Bassoon puncta intensity ( $129 \pm 5 \%$; $n=52$ ) when compared with neighboring untransfected neurons, the magnitude of its effect was significantly smaller than that of GFP-SAP97 ( $154 \pm 8 \% ; n=38$ ) (Fig. 2D). Expression of SAP102-GFP, on the other hand, caused only a very modest increase in average Bassoon intensity (109 $\pm 4 \% ; n=43)$, an increase that was much smaller than that of GFP-SAP97 expressed in sister cultures $(145 \pm 6 \% ; n=39)$ (Fig. $2 D$ ).

There are two possible explanations for the differences in the presynaptic effects of the postsynaptically expressed SAP97, PSD95, and SAP102: (1) intrinsic molecular differences in SAP97 make it more effective in enhancing presynaptic growth/maturation, or alternatively, (2) there is a direct correlation between the amount of postsynaptic MAGUK protein and presynaptic Bassoon content, and the observed differences in Bassoon staining are attributable to quantitative differences in the amount of recombinant protein at the synapses being assayed. That is, there are no intrinsic differences between the proteins in their abilities to enhance presynaptic growth/development. To address this latter possibility, we measured the level of GFP fluorescence at individual puncta as well as the intensity of the Bassoon puncta that specifically colocalized with these postsynaptically expressed GFP fusion proteins from stacked $z$-series confocal images. Figure $3 B$ shows that, as expected, the increase in Bassoon puncta intensity exclusively occurred at puncta that colocalized with postsynaptic GFP-SAP97, PSD-95-GFP, or SAP102-GFP and not at noncolocalized Bassoon puncta, which presumably reflect primarily inhibitory synapses on the transfected cells. Furthermore, consis- 


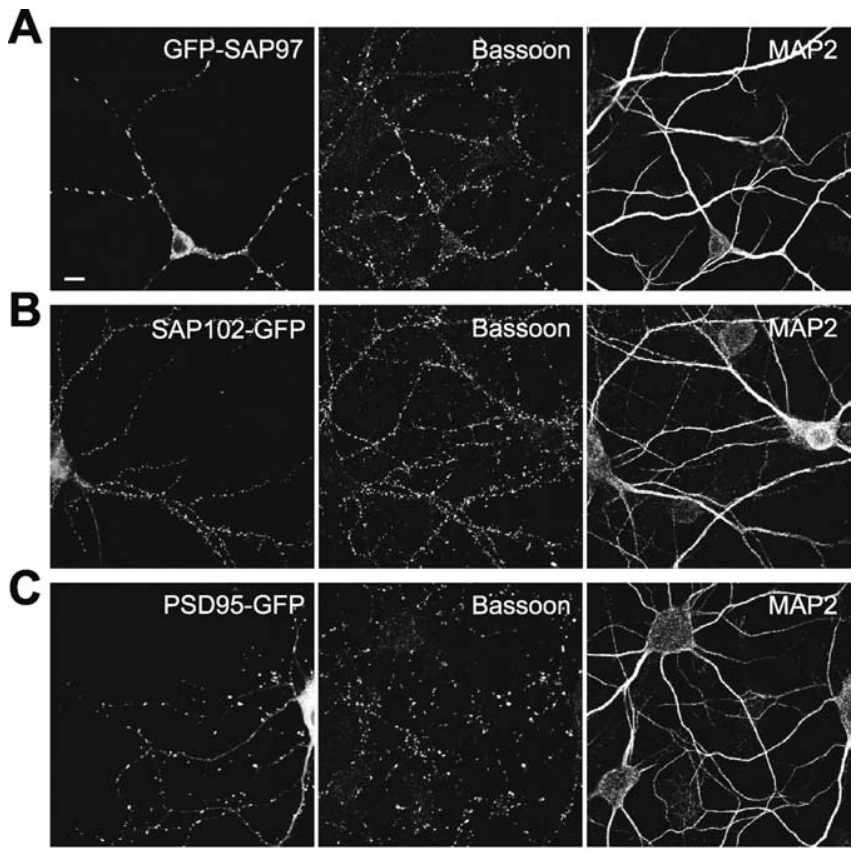

D

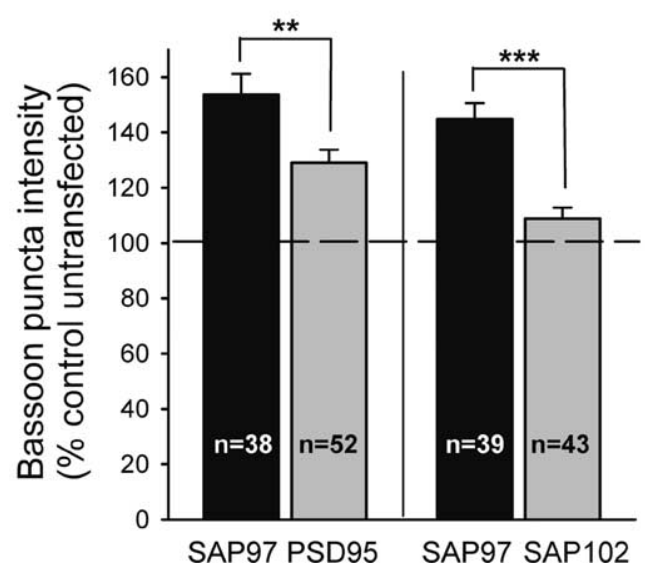

Figure 2. SAP97 has larger presynaptic effect than PSD-95 and SAP102. A-C, Representative images of hippocampal neurons transfected with GFP-SAP97 $(\boldsymbol{A})$, SAP102-GFP $(\boldsymbol{B})$, or PSD-95-GFP ( $C$ and double stained for Bassoon (middle) and MAP2 (right). Individual channels are shown in grayscale for better resolution. Scale bar: (in $A) 10 \mu \mathrm{m}$. D, Quantification of the effects of GFP-SAP97 and PSD-95-GFP (left part) or GFP-SAP97 and SAP102-GFP (right) on average Bassoon puncta intensity (mean $\pm \mathrm{SEM}$ ). Comparisons were made between transfected neurons from sister cultures ( $n=$ number of cells). ${ }^{* *} p<0.0005,{ }^{* *} p<0.005$; two-tailed $t$ test.

tent with the previous results, the increase in Bassoon puncta intensity was much larger for GFP-SAP97-expressing synapses $(252 \pm 12 \% ; n=53)$ than for synapses expressing PSD-95-GFP $(171 \pm 7 \% ; n=45)$ or SAP102-GFP $(131 \pm 7 \% ; n=36)$. Importantly, plotting the Bassoon intensity for individual puncta as a function of GFP fluorescence (Fig. 3C,D) revealed that the larger presynaptic effect of SAP97 was not because there was more SAP97 at individual synapses compared with PSD-95 or SAP102. That is, for a given amount of postsynaptically expressed MAGUK, SAP97 caused a significantly larger accumulation of presynaptic Bassoon. This was apparent when the fluorescence intensities for individual puncta were plotted (Fig. 3C,D, left graphs) as well as when an average ratio of Bassoon to GFP fluorescence for all puncta was calculated (Fig. 3C,D, right graphs). These results support the hypothesis that postsynaptic SAP97 promotes presynaptic terminal growth to a greater extent than
PSD-95 or SAP102, two other members of the same family of postsynaptic proteins.

\section{Postsynaptic SAP97 expression enhances presynaptic function}

Although the increased levels of presynaptic synaptophysin, synapsin, and Bassoon elicited by postsynaptic SAP97 expression are consistent with enlarged AZs and a greater number of synaptic vesicles at individual presynaptic terminals, these structural changes do not prove that a change in presynaptic function has occurred. Therefore, we examined the functional presynaptic effects of postsynaptic SAP97 expression using FM4-64 dye uptake, a well established method that allows labeling of the recycling pool of vesicles (Betz and Bewick, 1992; Ryan et al., 1993). FM4-64 fluorescence puncta intensity, a measurement that directly correlates with the number of actively cycling vesicles in each terminal, was increased almost twofold at puncta that colocalized with SAP97 when compared with puncta not expressing SAP97 on the same coverslips (SAP97 puncta, $1.88 \pm 0.08, n=$ 441; control puncta, $1.00 \pm 0.02, n=1776 ; p<0.0005$ ) (Fig. 4). Thus, as expected, the SAP97-induced changes in presynaptic protein content reflect a significant enhancement of presynaptic function.

Molecular determinants of the presynaptic effects of SAP97 To determine the role of specific SAP97 domains in mediating its enhancement of presynaptic terminal size, we examined the consequences of transfecting neurons with a series of GFP-tagged SAP97 deletion constructs. Although wild-type GFP-SAP97 routinely accumulated in dendritic puncta that colocalized with presynaptic markers, some deletion mutants displayed cell-to-cell variability in their distribution patterns ranging from relatively diffuse to highly punctate. We therefore limited our analysis to those cells in which clear GFP-SAP97 puncta that colocalized with Bassoon could be observed (for all examined constructs, this was a minimum of $40-45 \%$ of the transfected cells). Again, the increase in Bassoon staining occurred exclusively at puncta that colocalized with wild-type GFP-SAP97 $(220 \pm 10 \% ; n=80$ cells $)$ (Fig. $5 A, B$ ) or the different GFP-SAP97 constructs (Fig. $5 B$ ). Deletion of the $\mathrm{SH} 3$ domain, which was not necessary for efficient synaptic targeting of SAP97, did not significantly affect the increase in Bassoon staining ( $191 \pm 8 \% ; n=47$ ) (Fig. $5 B$ ). Similarly, deletion of the PDZ1 domain had no significant effect on the presynaptic effect of SAP97 or on its synaptic targeting (250 \pm $18 \% ; n=21$ ) (Fig. $5 B$ ). In contrast, expression of a construct lacking the S97N domain (amino acid residues 1-187) (SAP97 $\Delta$ S97N), which was still efficiently targeted to synapses, significantly decreased the enhancement of Bassoon staining caused by SAP97 by approximately one-half $(165 \pm 7 \% ; n=27)$ (Fig. $5 A, B$ ). Deleting the GK domain (SAP97 $\Delta \mathrm{GK}$ ) also robustly decreased the presynaptic effect of SAP97 $(146 \pm 7 \% ; n=25)$ (Fig. $5 A, B$ ) without significantly affecting its synaptic targeting. The largest effect was observed after deleting all three PDZ domains (SAP97 $\triangle \mathrm{PDZ1}-3$ ). This reduced the increase in Bassoon puncta intensity by $\sim 75 \%(128 \pm 6 \% ; n=28)$ (Fig. $5 A, B$ ), whereas deletion of the single PDZ3 domain (SAP97 $\triangle$ PDZ3) had a more modest effect $(172 \pm 7 \% ; n=45)$ (Fig. $5 B)$, comparable with that of deleting the S97N or GK domains. These results suggest that the SAP97-mediated enhancement of presynaptic terminal growth involves several of its protein-protein interaction domains with the PDZ domains being particularly important.

As discussed when comparing the presynaptic effects of 
SAP97 with PSD-95 and SAP102, it is possible that the reduction in the effects of the SAP97 deletion constructs on Bassoon puncta intensity may be attributable to a decrease in their levels at synapses relative to that of wild-type SAP97. To address that possibility, we again plotted Bassoon puncta intensity as a function of the amount of colocalized SAP97 (measured by the level of GFP fluorescence) for wildtype GFP-SAP97 and the two deletion constructs that showed the biggest effects, GFP-SAP97 $\triangle$ PDZ1-3 and GFPSAP97 $\Delta G K$. This analysis revealed that for a given amount of postsynaptic SAP97, wild-type SAP97 caused a larger increase in presynaptic terminal size than either of the two deletion constructs (Fig. 5C,D). This can be seen both by the differences in the linear regression lines that were fitted to the individual data points (left graphs) as well as the differences in the average ratio of Bassoon puncta intensity to GFP puncta intensity (right graphs). This analysis indicates that the smaller effect on Bassoon accumulation at individual synapses on neurons overexpressing these SAP97 deletion constructs is not caused by a decrease in their levels at synapses compared with wild-type SAP97. Instead, these results suggest that the enhancement of presynaptic active zone size by postsynaptic SAP97 specifically requires its PDZ and GK domains.

\section{Ligand binding to SAP97 PDZ domains} regulates presynaptic growth

It is conceivable that the relatively large deletions of SAP97 used in the previous experiments may have disrupted its tertiary structure and thereby impaired its ability to influence presynaptic bouton growth. Because the largest impairment was observed using SAP97 $\triangle$ PDZ1-3 and these PDZ domains have several suggested protein binding partners, which might signal transsynaptically such as neuroligin (Scheiffele, 2003; Kim and Sheng, 2004; Montgomery et al., 2004), we focused on generating SAP97 constructs with point mutations that disrupted the ligandbinding pockets of each PDZ domain (Doyle et al., 1996; Harris and Lim, 2001; Piserchio et al., 2002). Based on molecular modeling and structural studies, we chose to mutate the phenylalanine residue in the GLGF motif to histidine, a residue in-

volved in binding the C-terminal carboxylate group of group 1 PDZ ligands (Doyle et al., 1996; Songyang et al., 1997; Harris and Lim, 2001). For these studies, three sets of molecules were generated: single point mutants in each individual $\mathrm{PDZ}$ domain $\left(\mathrm{F}_{203} \rightarrow \mathrm{H}\right.$ in $\mathrm{PDZ} 1, \mathrm{~F}_{298} \rightarrow \mathrm{H}$ in $\mathrm{PDZ} 2$, and $\mathrm{F}_{470} \rightarrow \mathrm{H}$ in $\left.\mathrm{PDZ3}\right)$, double point mutants in two of the three PDZ domains, and a

A

C
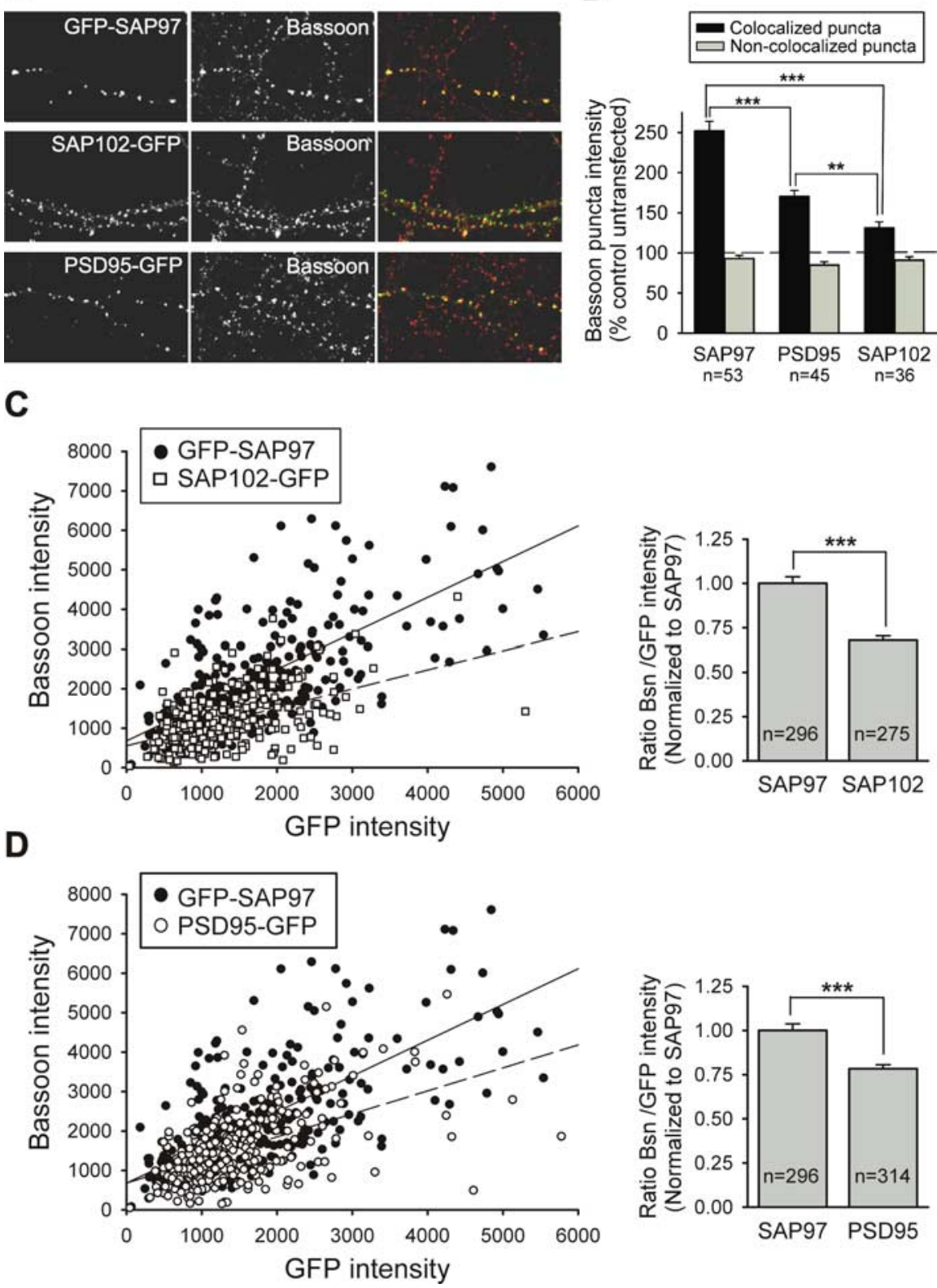

Figure 3. Differences in the presynaptic effects of SAP97, PSD-95, and SAP102 are not attributable to differences in their synaptic targeting. $\boldsymbol{A}$, Representative images of neurons transfected with GFP-SAP97, SAP102-GFP, or PSD-95-GFP as indicated. GFP fluorescence is shown in the left panels, Bassoon staining is shown in the middle panels, and merged images are shown in the right panels. B, Quantification of the average Bassoon puncta intensity induced by overexpression of GFP-SAP97, SAP102-GFP, or PSD-95-GFP. The black bars show the average intensity of Bassoon puncta that colocalize with GFP-tagged proteins in a single dendrite, and the gray bars show the average intensity of Bassoon puncta that do not colocalize in the same dendrite (mean \pm SEM; $n=$ number of cells). ${ }^{* * *} p<0.0005,{ }^{* *} p<0.005$; one-way ANOVA test and a Tamhane post test. $\boldsymbol{C}$, Left, Intensities of individual Bassoon (Bsn) puncta are plotted as a function of the corresponding colocalized GFP-SAP97 or SAP102-GFP puncta intensities (4 transfected cells each). Linear regression is shown as a solid line for GFP-SAP97 and a dashed line for SAP102-GFP. Right, Comparison of Bassoon/GFP intensity ratio for individual puncta expressing GFP-SAP97 or SAP102-GFP. Comparisons were made between transfected neurons from sister cultures. Data were normalized to Bsn/GFP-SAP97 ratio (mean \pm SEM; $n=$ number of puncta; same data as in left panel). ${ }^{* * *} p<0.0005$; two-tailed $t$ test. $\boldsymbol{D}$, Same as $\boldsymbol{C}$ except data from PSD-95-GFPtransfected cells $(n=5)$ are plotted.

triple PDZ point mutant. To test the efficacy of these mutations, we performed GST pull-down assays to examine the ability of the full-length double point or triple point mutant constructs to bind to GST-fusion versions of either: (1) the C-terminal tail of NR2B containing its PDZ-binding domain, which has been reported to interact with a SAP97 construct containing PDZ1 and 2 (Ni- 
A
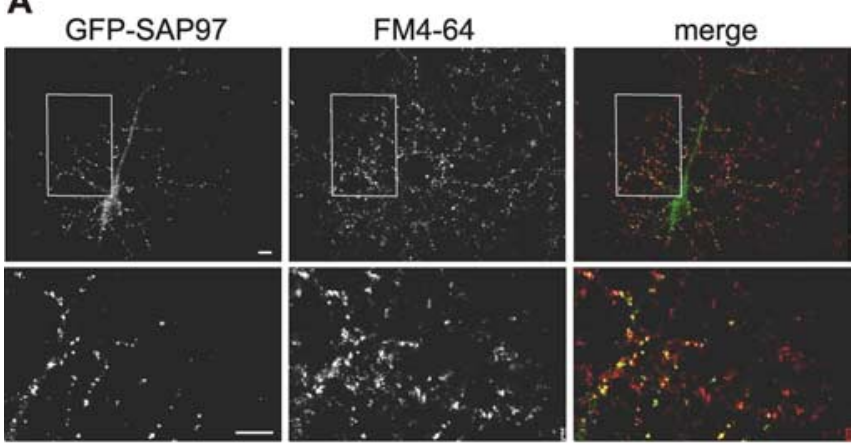

B

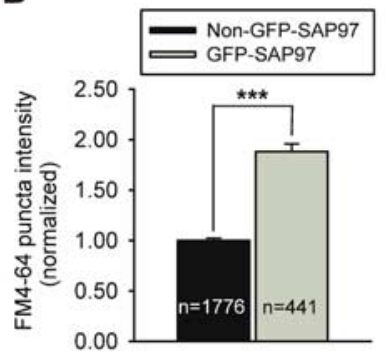

C

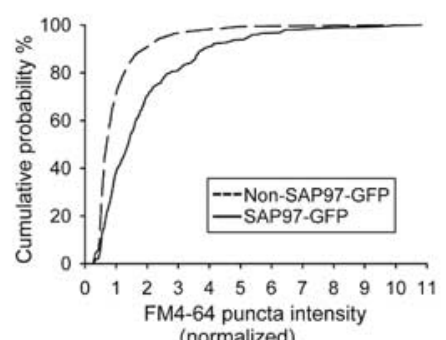

(normalized)

Figure 4. Postsynaptic SAP97 expression enhances presynaptic function. $\boldsymbol{A}$, Representative image of FM4-64 staining of functional presynaptic terminals on neurons transfected with GFP-SAP97. GFP fluorescence is shown in the left panels, FM4-64 fluorescence is shown in the middle panels, and merged images are shown in the right panels. Individual channels are shown in grayscale for better resolution. The bottom panels are higher-magnification images of the boxed regions in the top panels. Scale bars, $10 \mu \mathrm{m}$. B, Quantitative analysis of changes in average FM4-64 puncta intensity comparing puncta colocalized with GFP-SAP97 (gray bar) versus non-colocalized, control puncta (black bar) on the same coverslips. Bars show mean \pm SEM normalized to the average control puncta intensity $\left(n=\right.$ number of puncta; ${ }^{* *} p<$ 0.0005 , two-tailed $t$ test). C, Cumulative probability distribution of FM4-64 puncta intensities (same data as in $\boldsymbol{B}$ ).

ethammer et al., 1996) or (2) the segment of CRIPT containing its PDZ-binding domain, which originally was reported to interact primarily with the PDZ3 domain of PSD-95 and SAP97 (Niethammer et al., 1998; Cai et al., 2002), but we (see below) and others (Lim et al., 2002; Piserchio et al., 2002; Long et al., 2003) find binds all three PDZ domains. The GST-NR2B C-tail exhibited robust binding to wild-type GFP-SAP97 as well as the double point mutants containing mutations in PDZ1 and 3 (GFPSAP97PDZ1\&3FH) or PDZ2 and 3 (GFP-SAP97PDZ2\&3FH) (supplemental Fig. 1, available at www.jneurosci.org as supplemental material). However, GFP-SAP97PDZ1\&2FH, in which both PDZ1 and 2 contain point mutations, showed almost no binding to the NR2B C-tail, indicating that the $\mathrm{F} \rightarrow \mathrm{H}$ mutations inactivated the ligand binding pocket of these two PDZ domains. In analogous experiments with the C-terminal of CRIPT, we found that GST-CRIPT robustly bound wild-type GFP-SAP97 as well as GFP-SAP97PDZ1\&2FH but failed to bind GFP-SAP97 with point mutations in all three PDZ domains (GFPSAP97PDZ123FH) (supplemental Fig. 1, available at www.jneurosci.org as supplemental material). These data verify that the $\mathrm{F} \rightarrow \mathrm{H}$ mutation also inactivates ligand binding to the PDZ3 domain.

Having confirmed the efficacy of the point mutants in preventing ligand binding, we examined their ability to enhance presynaptic active zone growth as measured by Bassoon staining. None of the single point mutants in PDZ1-3 had a significant effect on the enhancement of Bassoon puncta intensity when compared with wild-type SAP97 (wild-type GFP-SAP97, 236 土 $7 \%, n=120$; GFP-SAP97PDZ1F203H, $243 \pm 14 \%, n=29$; GFP-SAP97PDZ2F298H, $224 \pm 17 \%, n=20$; GFPSAP97PDZ3F470H, $210 \pm 21 \%, n=18)(6 A, B)$. However, expression of any combination of double point mutants significantly decreased the normal increase in Bassoon puncta intensity by at least one-half (GFP-SAP97PDZ1\&2FH, $159 \pm 15 \%, n=$ 19; GFP-SAP97PDZ1\&3FH, $156 \pm 10 \%, n=13$; GFPSAP97PDZ2\&3FH, $145 \pm 10 \%, n=26$ ) (Fig. 6A,B). We also tested the triple mutant GFP-SAP97PDZ123FH, but it appeared to be toxic to the cells.

To examine whether the reduction in the effect on presynaptic terminal growth of these SAP97 point mutants might be caused by a relative decrease in their synaptic expression levels compared with wild-type SAP97, we compared individual Bassoon puncta intensity with the colocalized GFP fluorescence. This analysis revealed that point mutations in PDZ1 and 2 had the largest effect, causing a clear decrease in the slope of the linear regression line when individual puncta were plotted and, on average, a $43 \%$ decrease in Bassoon puncta intensity for a given amount of postsynaptic SAP97 (wild-type SAP97, $1.0 \pm 0.05, n=163$; SAP97PDZ1\&2FH, $0.57 \pm 0.03, n=182$ ) (Fig. 6C1). Point mutations in PDZ1 and 3 also decreased the normal presynaptic effect of SAP97, although the magnitude of this inhibition was significantly smaller than that of PDZ1 and 2 mutations (wildtype SAP97, $1.0 \pm 0.03, n=314$; SAP97PDZ1\&3FH, $0.92 \pm 0.03$, $n=295$ ) (Fig. 6C2). These results suggest that the interaction of SAP97 with primarily its PDZ1 and PDZ2 binding partners are the most important for its ability to promote presynaptic terminal growth.

Surprisingly, point mutations in PDZ2 and 3 had minimal effects on the increase in Bassoon staining caused by GFP-SAP97 when normalized to the amount of postsynaptic SAP97 (wildtype SAP97, $1.0 \pm 0.04, n=205$; SAP97PDZ2\&3FH, $0.90 \pm 0.04$, $n=161$ ) (Fig. 6C3). One possible explanation for this result is that this double mutation decreased the amount of recombinant SAP97 at synapses compared with wild-type SAP97. The average GFP intensity at individual puncta for GFP-SAP97PDZ2\&3FH $(1190.35 \pm 62.67 ; n=161)$ was in fact significantly less than that of GFP-SAP97 (1493.47 $\pm 76.34 ; n=205 ; p<0.01)$.

Together, these results support our deletion analysis indicating that the PDZ domains of SAP97 play an essential role in transducing a transsynaptic signal to the presynaptic active zone. Furthermore, they indicate that whereas ligands for PDZ1 and PDZ2 are the most important, ligands docking at PDZ3, such as neuroligin 1 (Irie et al., 1997), may not be critically involved (see below). It is important to note that whereas mutating the ligand binding pocket of PDZ3 had no affect on the increase in Bassoon staining, deleting the entire PDZ3 domain (SAP97 $\triangle$ PDZ3) (Fig. 5 ) caused a significant reduction in Bassoon staining. These data suggest that some aspect of PDZ3 function other than ligand binding, perhaps its phosphorylation (Sabio et al., 2005), may also be important for SAP97-induced enhancement of presynaptic active zone growth.

\section{SAP97 recruitment of other postsynaptic proteins}

Given its multiple protein-protein interaction domains, it is likely that GFP-SAP97 recruited other postsynaptic proteins that may have contributed to the observed enhancement of presynaptic terminal growth. We therefore used immunocytochemical assays to examine changes in the levels of a number of postsynaptic proteins that interact directly or indirectly with SAP97 and have been reported to have effects on the maturation/growth of syn- 
A
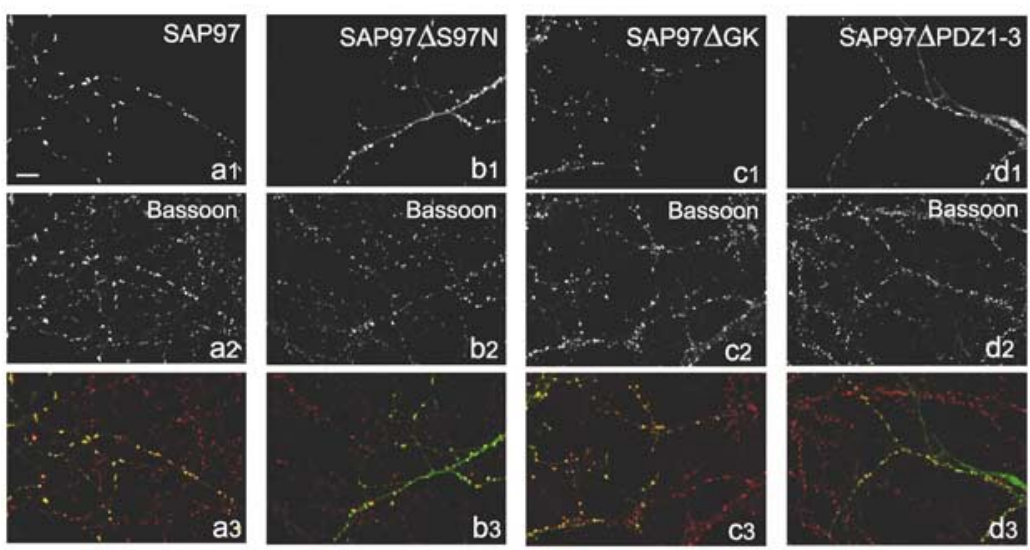

B
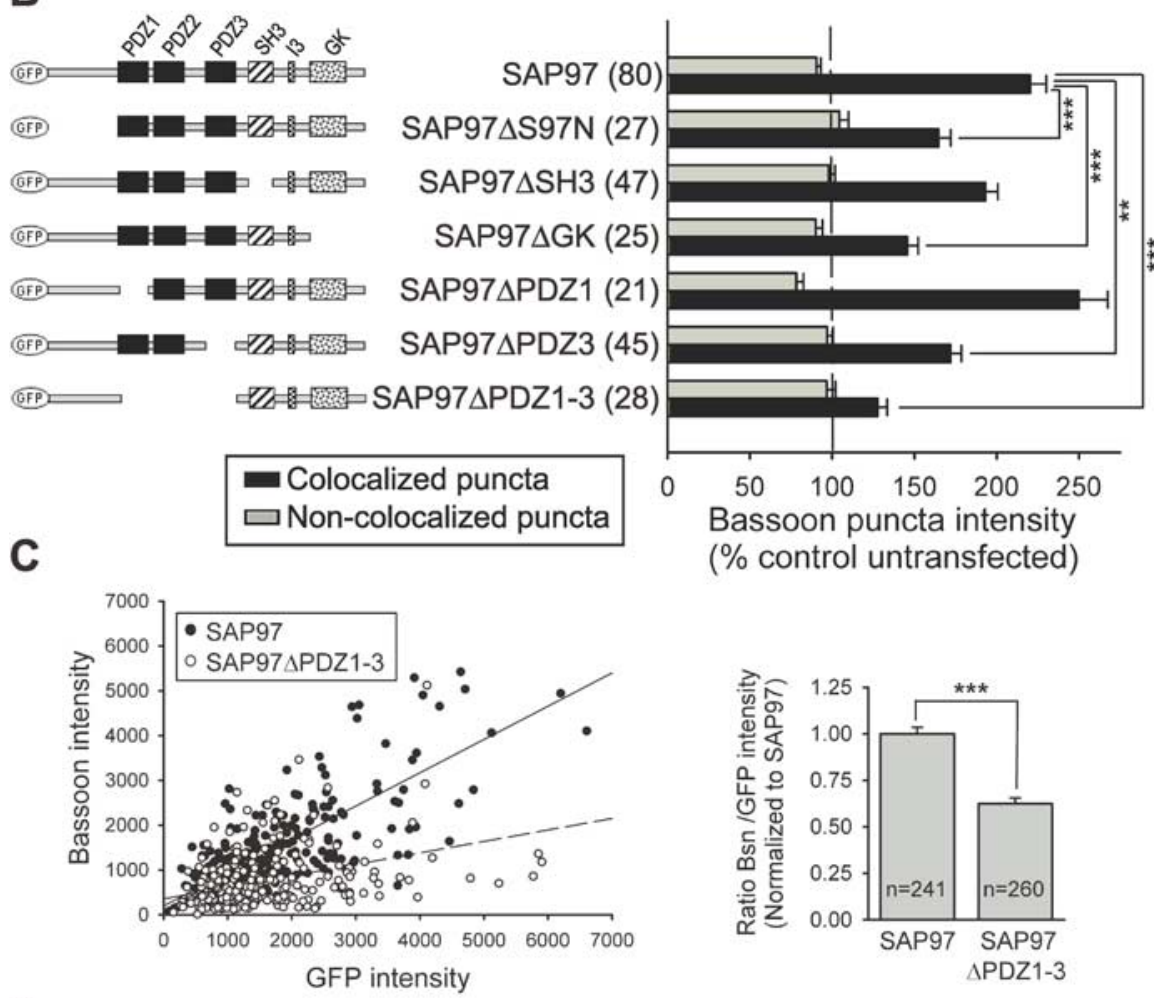

D
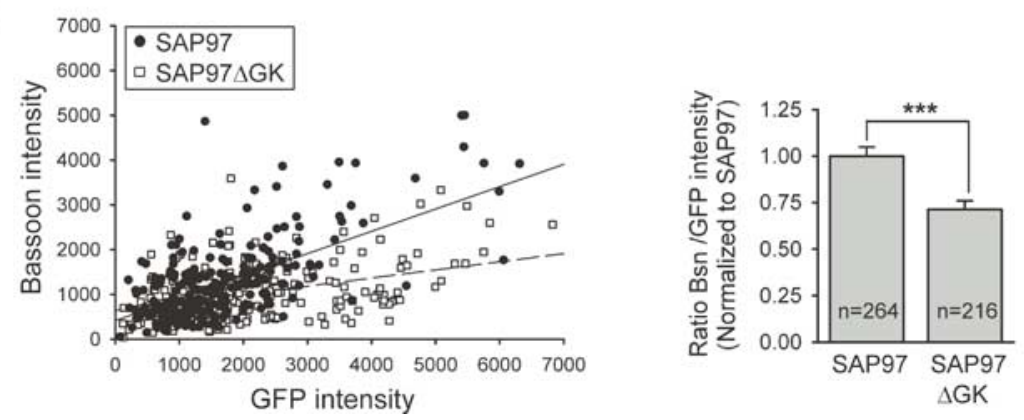

Figure 5. Specific structural domains of $S A P 97$ are required for its presynaptic effects. $A$, Representative images of neurons transfected with GFP-SAP97 (a1), GFP-SAP97 SS97N (b1), GFP-SAP97 GGK (c1), and GFP-SAP97 DPDZ1-3 (d1). Each neuron was double stained for Bassoon ( $(\mathbf{a} 2-\boldsymbol{d 2}$ ) and MAP2 (not shown). Merged images are shown in $\boldsymbol{a} \mathbf{3}-\mathbf{d}$ 3. Scale bar: (in a1) $10 \mu \mathrm{m}$. $B$, Left, Schematic diagrams of SAP97 constructs used in this study. Different structural domains are indicated. Right, Quantification of changes in Bassoon puncta intensity induced by the different SAP97 constructs. The black bars show the average intensity of Bassoon puncta that colocalize with the GFP-SAP97 puncta in a single dendrite, and the gray bars show the average intensity of puncta that do not colocalize in the same dendrite (mean \pm SEM; numbers in parentheses indicate number of cells examined). Neurons expressing GFP-SAP97 SS97N, GFP-SAP97DGK, GFP-SAP97 DPDZ3, or GFP-SAP97DPDZ1-3 showed a significant decrease in the average Bassoon puncta intensity when compared with neurons overexpressing wild-type GFP-SAP97 $\left({ }^{* * *} p<\right.$ $0.0005,{ }^{* *} p<0.005$; one-way ANOVA test and a Tamhane post test). C, Left, Intensities of individual Bassoon puncta are plotted apses and dendritic spines. For example, Shank1a and ProSAP2 (also known as Shank3) are members of a family of PDZdomain-containing proteins that interact directly or indirectly with receptors of the postsynaptic membrane, including NMDA receptors (NMDARs) and metabotropic glutamate receptors, and the actin cytoskeleton (Sheng and Kim, 2000; Boeckers et al., 2002). Both Shank1a and ProSAP2/Shank3 use the GK domain binding protein GKAP to associate with members of the SAP90/PSD-95 family, including SAP97 (Boeckers et al., 1999; Naisbitt et al., 1999). Furthermore, Shank1a and ProSAP2/Shank3 have been reported to lead to dendritic spine enlargement and enhancement of presynaptic function (Sala et al., 2001), similar to SAP97 (Rumbaugh et al., 2003). Consistent with a possible role for these proteins in mediating the effects of SAP97, cells expressing GFP-SAP97 showed $\sim 60 \%$ increase in ProSAP2 and Shank1a puncta intensity compared with adjacent untransfected neurons (Fig. 7B,E,G). Similarly, the levels of postsynaptic SPAR/ SPAL, a RapGAP for Rap1, which promotes integrin mediated cell-cell adhesion (Roy et al., 2002), interacts with the GK domain of SAP97 and SAP90/PSD-95, and enhances spine growth when overexpressed in cultured neurons (Pak et al., 2001; Roy et al., 2002), was also increased by $57 \%$ in neurons expressing GFPSAP97 (Fig. $7 F, G$ ). Consistent with previous work (Rumbaugh et al., 2003), we also found that expression of GFP-SAP97 increased the levels of GluR1 at synapses (Fig. 7C,G) when compared with untransfected cells.

GFP-SAP97 had much smaller effects on the postsynaptic levels of PSD-95 and Homer1b,c, which were only modestly increased by 17 and 22\%, respectively (Fig. $7 A, D, G)$. The latter result was surprising given that Homer interacts directly with Shank and is required for mediating the effects of Shank on spine morphology

\section{$\leftarrow$}

as a function of the corresponding colocalized GFP-SAP97 or GFP-SAP97DPDZ1-3 puncta intensities (9 transfected cells each). Linear regression is shown as a solid line for GFP-SAP97 and a dashed line for SAP97 $\triangle P D Z 1-3$. Right, Comparison of Bassoon (Bsn)/GFP intensity ratio for individual puncta expressing GFP-SAP97 or SAP97DPDZ1-3. Comparisons were made between transfected neurons from sister cultures. Data were normalized to the Bsn/GFP-SAP97 ratio (mean \pm SEM; $n=$ number of puncta; same data as in left panel). ${ }^{* *} p<$ 0.0005 , two-tailed $t$ test. D, Same as $C$, except data from GFPSAP97- and GFP-SAP97 $\triangle$ GK-transfected cells (5 and 7 cells, respectively) are plotted. 
A
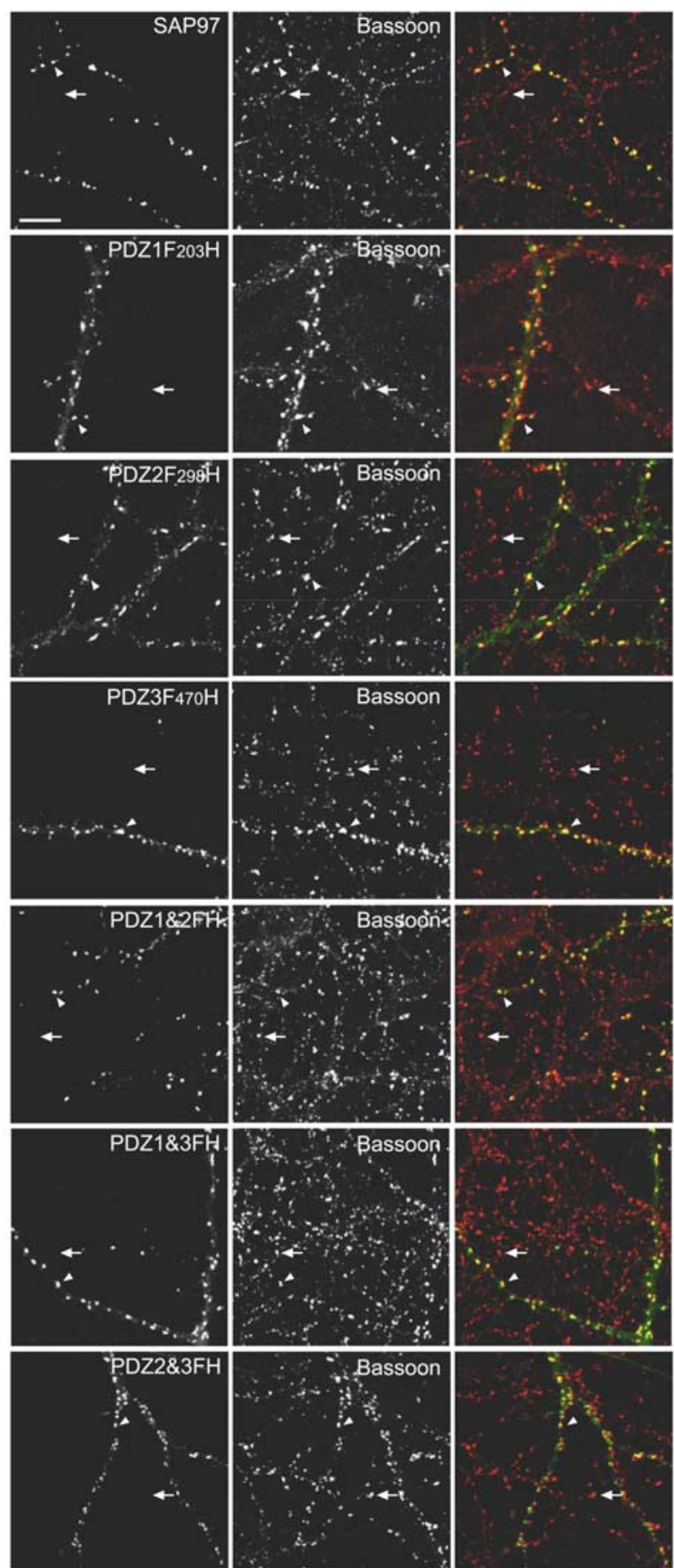

C

\section{C1}
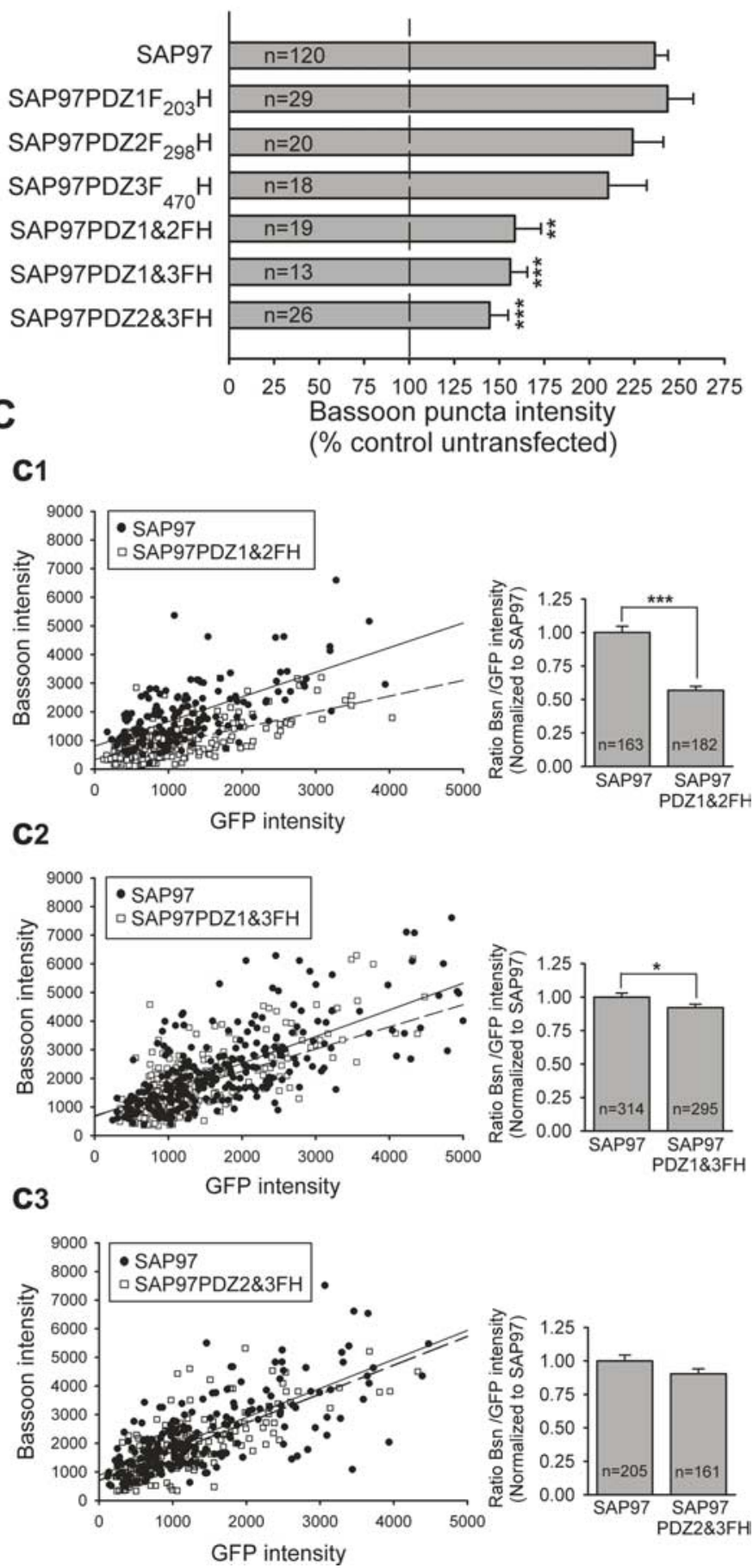

Figure 6. SAP97 PDZ1 and PDZ2 ligand-binding pockets are critical for its presynaptic effects. $A$, Representative images of neurons transfected with GFP-SAP97 or GFP-SAP97 PDZ domain mutants, as indicated in the left panels. Each row of images shows GFP fluorescence (left), Bassoon labeling (middle), and merged images (right). Staining for MAP2 is not shown. The arrowheads indicate Bassoon puncta on transfected neurons. The arrows indicate Bassoon puncta on untransfected neighboring neurons. Scale bar, $10 \mu \mathrm{m}$. Mutants are point mutants for the ligand-binding

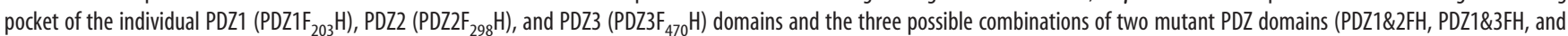
PDZ2\&3FH). B, Quantification of the average Bassoon puncta intensity that colocalized with puncta of the GFP-tagged SAP97 mutants (mean \pm SEM; $n=$ number of cells; ${ }^{* * *} p<0.0005,{ }^{* *} p<$ 0.005 , one-way ANOVA test and a Tamhane post test). Neurons expressing any of the double PDZ domain point mutants, PDZ1\&2FH, PDZ1\&3FH, and PDZ2\&3FH, showed a significant decrease in the average Bassoon puncta intensity when compared with neurons expressing wild-type GFP-SAP97. C, Left, Intensities of individual Bassoon puncta are plotted as a function of the corresponding colocalized GFP-SAP97 or GFP-SAP97PDZ1\&2FH (C1), GFP-SAP97PDZ1\&3FH (C2), and GFP-SAP97PDZ2\&3FH (C3) ( $n=3$ or 4 transfected cells each). Linear regression is shown as solid lines for GFP-SAP97 and dashed lines for the mutant GFP-SAP97s. Right, Comparison of Bassoon (Bsn)/GFP intensity ratio for individual puncta expressing GFP-SAP97 or the PDZ mutants. Comparisons were made between transfected neurons from sister cultures. Data were normalized to the Bsn/GFP-SAP97 ratio (mean \pm SEM; $n=$ number of puncta; same data as in left panels; ${ }^{* * *} p<0.0005$, ${ }^{*} p<0.05$, two-tailed $t$ test). 
(Sala et al., 2001). However, given that Homer only increased when Shank reached it highest levels (Sala et al., 2001) and has not been tested for a role in transducing the presynaptic effects of Shank, this discrepancy may represent divergent properties of Shank and Homer. Together, our results suggest that increasing the postsynaptic levels of SAP97 leads to the selective recruitment of specific postsynaptic proteins, several of which, including SPAR/SPAL and Shank/ProSAP, may indirectly induce the growth/ maturation of presynaptic terminals.

Both SAP97 and PSD-95 can recruit AMPARs to synapses (Schnell et al., 2002; Rumbaugh et al., 2003; Nakagawa et al., 2004), although perhaps via distinct mechanisms. SAP97 can directly interact with GluR1 (Leonard et al., 1998), whereas PSD-95 interacts with GluR1 via stargazin (Schnell et al., 2002). It was therefore of interest to directly compare under identical experimental conditions the ability of the two MAGUK family members to increase GluR1 at synapses, especially given that SAP97 had larger effects on presynaptic growth than PSD-95 (Figs. 2, 3). Surprisingly, we found that GFP-SAP97 and PSD-95-GFP had similar effects on GluR1 puncta intensity (SAP97, $184 \pm 18 \%, n=20$; PSD-95, $163 \pm 14 \%, n=23$ ) (Fig. 8 ).

\section{Role of transsynaptic proteins in SAP97-mediated presynaptic terminal growth}

The enhanced levels of active zone and synaptic vesicle proteins in presynaptic axon terminals contacting neurons expressing GFP-SAP97 must be attributable to some retrograde signal from the postsynaptic cell back to the terminals. Prime candidates for subserving this function are transsynaptic cell adhesion molecules, which have been suggested to play a role in synapse development and maturation, including the integrins, cadherins, and neuroligins as well as the Eph receptor tyrosine kinases and their intercellular binding partners, the ephrin ligands. (Tang et al., 1998; Zhai et al., 2000; Chavis and Westbrook, 2001; Contractor et al., 2002; Garner et al., 2002; Li and Sheng, 2003; Scheiffele, 2003). To investigate the role of these proteins in mediating the presynaptic effects of postsynaptic SAP97, we chronically treated cultures with reagents that inhibit their transsynaptic interactions. For example, chronic treatment of GFP-SAP97-transfected cells with GRGDSP, a peptide that disrupts interactions mediated by integrins (Ruoslahti, 1996; Chavis and Westbrook, 2001), caused a $46 \%$ reduction in the increase of Bassoon puncta intensity when compared with untreated neurons expressing GFP-SAP97 (Fig.
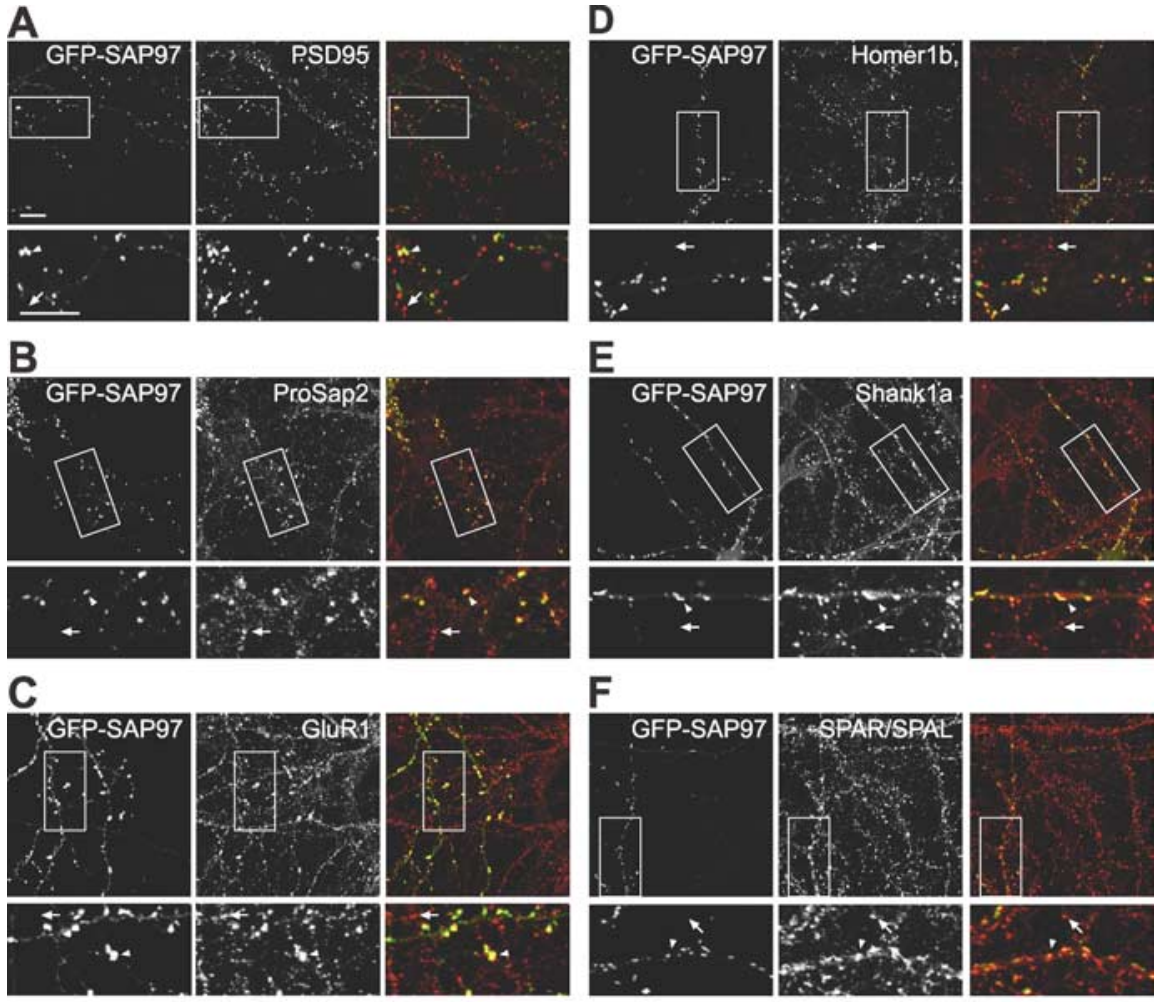

$\mathbf{F}$
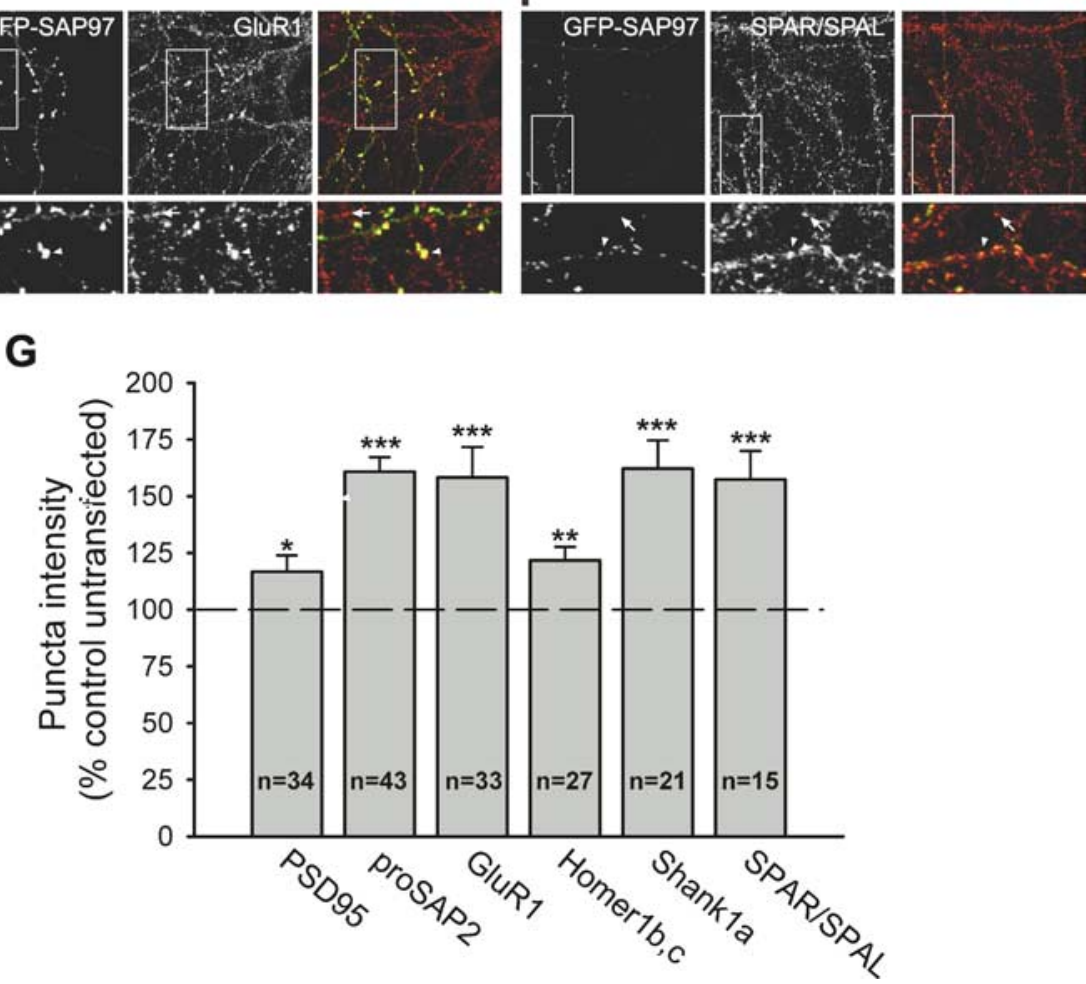

Figure 7. SAP97 recruits additional postsynaptic proteins. $\boldsymbol{A}-\boldsymbol{F}$, Representative images of neurons transfected with GFPSAP97 and stained for PSD-95 (A), ProSAP2 (B), GluR1 (C), Homer1b,C (D), Shank1a $(\boldsymbol{E})$, and SPAR/SPAL $(\boldsymbol{F})$. The left panels show GFP fluorescence, the middle panels show the immunostained protein, and the right panels show merged images. The bottom panels are higher-magnification images of the boxed regions in the top panels. Scale bars: (in $A$ ) $10 \mu \mathrm{m}$ ). The arrowheads indicate puncta colocalized with GFP-SAP97 on transfected cells; the arrows indicate puncta on untransfected neighboring neurons. $\mathbf{G}$, Quantification of the average puncta intensity of the indicated endogenous proteins in neurons expressing GFP-SAP97. The histograms show mean \pm SEM expressed as percentage of average puncta intensity in neighboring untransfected neurons in the same field ( $n=$ number of cells; ${ }^{* * *} p<0.0005,{ }^{* *} p<0.005,{ }^{*} p<0.05$, two-tailed $t$ test).

$9 A, B)$. Two other manipulations caused similar inhibition of the SAP97-mediated presynaptic active zone growth: (1) inhibition of $\mathrm{N}$-cadherin homophilic interactions using a functionblocking antibody (Tang et al., 1998) (Fig. 9A,B) and (2) inhibition of transsynaptic EphB receptor-ephrinB signaling using dimers of the extracellular domain of ephrin-B1 ligands fused to the $\mathrm{Fc}$ region of human IgG (Ephrin-B1-Fc), which act primarily as blocking agents (Dalva et al., 2000; Contractor et al., 2002) 
A
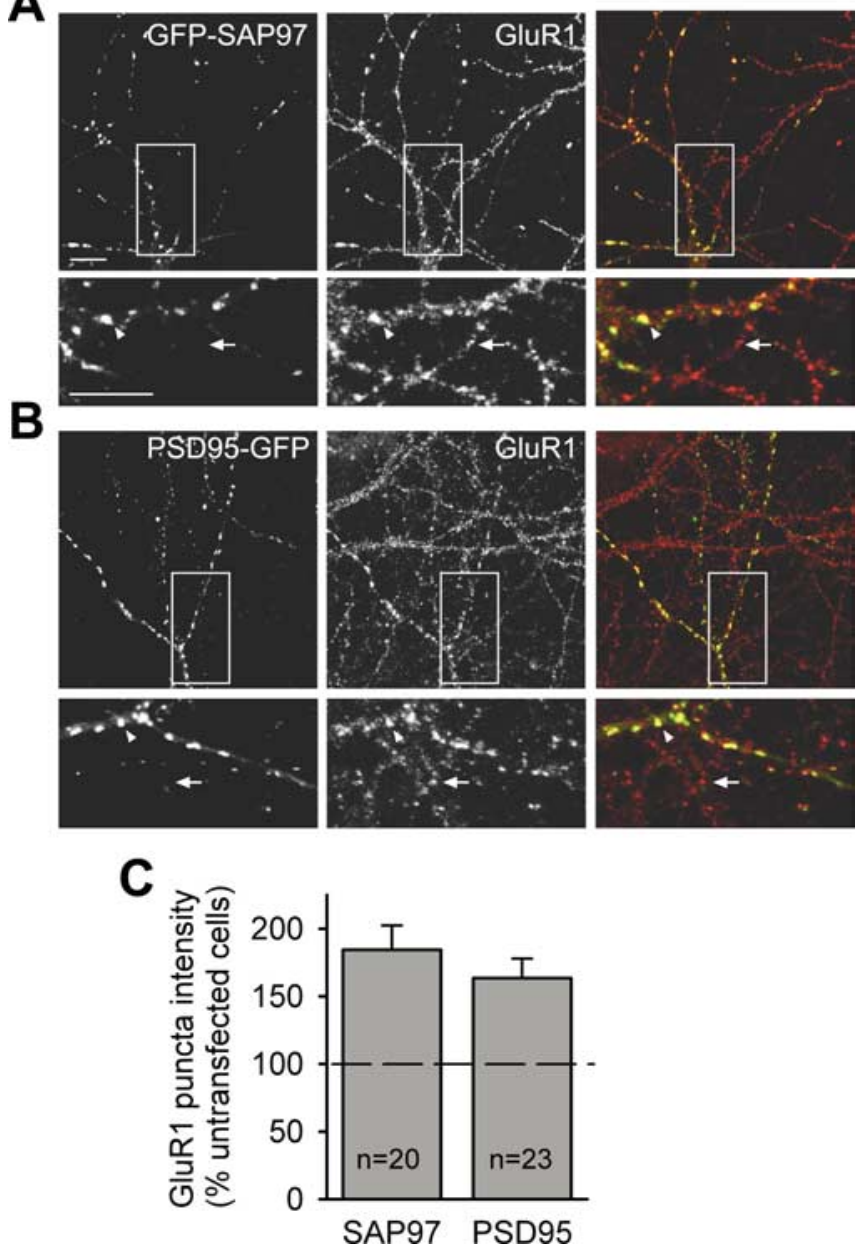

Figure 8. SAP97 and PSD-95 both enhance surface expression of AMPA receptors. $\boldsymbol{A}, \boldsymbol{B}$, Representative images of neurons transfected with GFP-SAP97 (A) or PSD-95-GFP $(\boldsymbol{B})$ and immunostained for surface GluR1. The left panels show GFP fluorescence, the middle panels show GluR1 surface staining, and the right panels show merged images. The bottom panels are higher-magnification images of the boxed regions in the top panels. Scale bars: (in $\boldsymbol{A}$ ) $10 \mu \mathrm{m}$. The arrowheads indicate GluR1 puncta colocalized with GFP-SAP97 in transfected neurons; the arrows indicate GluR1 puncta on untransfected neighboring neurons. C, Quantification of the effect of GFP-SAP97 and PSD-95-GFP expression on the average surface GluR1 puncta intensity. The histograms show mean \pm SEM expressed as percentage of average puncta intensity in neighboring untransfected neurons in the same field ( $n=$ number of cells).

(Fig. 9A,B). As a control, cells were treated with IgG $(\mathrm{Fc})$ alone, and this had no effect on the increase in Bassoon puncta intensity caused by GFP-SAP97 (untreated, $214 \pm 17 \%, n=16$; IgG treated, $225 \pm 25 \%, n=13$; data not shown).

The soluble form of $\beta$-neurexin has also been reported to interfere with the development of presynaptic terminals mediated by neuroligin-neurexin transsynaptic signaling (Scheiffele et al., 2000). Surprisingly, we observed no inhibitory effect of chronic treatment with a soluble form of $\beta$-neurexin that contained its extracellular domain fused to $\mathrm{Fc}(\beta$-neurexin-Fc) (Fig. $9 A, B)$. This finding is consistent with a minimal role for the PDZ3 domain in SAP97, a likely site of neuroligin interaction (Irie et al., 1997), in the induction of transsynaptic changes. Likewise, treatment with a soluble form of Ephrin-A5 containing its extracellular domain fused to Fc (EphrinA5-Fc) (Lawrenson et al., 2002) did not affect the increase in Bassoon accumulation elicited by GFP-SAP97 (Fig. 9B).
It is conceivable that inhibiting transsynaptic protein interactions may have destabilized SAP97 at synapses, and this may have contributed to the decrease in Bassoon accumulation. However, for a given amount of postsynaptic SAP97, assessed by GFP fluorescence, the Bassoon puncta intensity was still clearly decreased by the treatments with inhibitors of N-cadherins, integrins, or EphB receptor/EphrinB1. This can be seen in the plots of Bassoon versus GFP intensity for individual puncta (Fig. 9C) as well as by the average ratio of Bassoon to GFP intensity (Fig. 9D) for the different treatments.

Together, these results suggest that a number of transsynaptic proteins including $\mathrm{N}$-cadherins, integrins, and EphB receptor/ EphrinB1 participate in transducing a presynaptic terminal growth signal in response to increases in postsynaptic SAP97. This transsynaptic signal appears to be specific as the induction was unaffected by the addition of either $\beta$-neurexin-Fc or ephrinA5-Fc. To examine whether cadherin, integrin, and Ephrin/EphB cooperate in transducing this signal, we applied inhibitors of all three systems together. This combination had no greater effect on inhibiting presynaptic terminal growth $(\sim 50 \%$ reduction) than any one alone (Fig. $9 A, B$ ), suggesting that all three transsynaptic protein interactions converge on the same mechanisms presynaptically and that SAP97 may activate additional transsynaptic signaling pathways that work in parallel.

\section{Discussion}

The molecular mechanisms by which the tight registration between postsynaptic PSDs and presynaptic AZs are maintained during synapse maturation and growth are primarily unknown. To explore these mechanisms, we examined the presynaptic consequences of overexpressing members of the MAGUK family of postsynaptic scaffolding proteins. We found that postsynaptic expression of SAP97 stimulates synapse formation and promotes the accumulation of several endogenous presynaptic proteins including synaptophysin, a synaptic vesicle protein, and Bassoon, a core component of the active zone. Although accumulation of presynaptic proteins does not prove that increases in the sizes of presynaptic boutons/active zones have occurred, previous studies have shown that increased synaptophysin immunoreactivity correlates with an increase in synaptic bouton vesicle content (Belichenko et al., 2004). Moreover, the Bassoon increases are consistent with the role of Bassoon in quantal growth of active zones during synapse formation (Shapira et al., 2003). Importantly, postsynaptic SAP97 expression enhanced presynaptic function, as evidenced by increased FM4-64 staining. Thus, these immunocytochemical and functional changes are consistent with postsynaptic SAP97 inducing presynaptic terminal growth.

SAP97 promoted presynaptic terminal growth to a greater extent than other family members, specifically PSD-95 and SAP102. This was not because of differences in the amount of the recombinant proteins present at individual synapses, suggesting that small structural differences in family members can translate into important functional differences. The larger effect of SAP97 is consistent with studies showing that SAP97 more efficiently rescued defects at the neuromuscular junction in Drosophila dlg mutant larvae than either PSD-95 or SAP102 (Thomas et al., 1997). Our results are also consistent with an electrophysiological study that suggested that SAP97 may have a broader role in synapse growth and/or maintenance than PSD-95 (Nakagawa et al., 2004).

What features in SAP97 account for its greater effect on presynaptic terminal growth? One possibility is that although all family members contain structurally related protein interaction 
domains, their specific binding partners differ. A second is that "uniqueness" is encoded by sequences flanking these conserved domains. Regarding the former, numerous in vitro binding studies suggest that, in general, the PDZ, SH3, and GK domains share similar binding specificities (Montgomery et al., 2004). One exception is that the PDZ1 domain of SAP97 appears to exhibit remarkable specificity for the C-terminal tails of AMPAR GluR1 subunits (Leonard et al., 1998; Cai et al., 2002). It seems unlikely, however, that this property of SAP97 accounts for its larger presynaptic effects as both SAP97 and PSD-95 had similar effects on synaptic GluR1 accumulation (Fig. 8). Arguably the most novel features of SAP97 are its $\mathrm{N}$-terminal domain (S97N) and the hook region situated between the $\mathrm{SH} 3$ and GK domains (Wu et al., 2000). The S97N domain enables SAP97 to form multimers (Marfatia et al., 2000; Nakagawa et al., 2004) and bind cytoskeletal proteins such as CASK (Lee et al., 2002) and myosin VI (Wu et al., 2002). By homomultimerization with itself or heteromultimerization with other proteins, SAP97 may build postsynaptic multimolecular complexes more effectively than other family members such as PSD-95, and this in turn may promote presynaptic growth more effectively.

The SAP97 hook region is important because it is a site of alternative splicing (Montgomery et al., 2004) and appears to affect the spatial distribution of SAP97 (McLaughlin et al., 2002; Rumbaugh et al., 2003). The SAP97 splice variant used in these studies contains an I3 insert, which has been shown to direct the postsynaptic localization of SAP97 (Rumbaugh et al., 2003), presumably through its ability to bind the actin/spectrin cytoskeletal protein 4.1 (Lue et al., 1994; Hanada et al., 2003; Rumbaugh et al., 2003). This interaction may be relevant because neither PSD-95 nor SAP9102 have an I3 like sequence nor can they bind protein 4.1. Furthermore, in contrast to PSD-95, this interaction appears to place SAP97 in a different subsynaptic compartment that is sensitive to the disassembly of filamentous actin (Rumbaugh et al., 2003).

Experiments using deletion constructs and point mutants revealed that three domains of SAP97 were particularly critical for mediating its presynaptic effects: the S97N domain, the first two PDZ domains, and the GK domain. As mentioned above, the S97N domain may be important because of its ability to multimerize and/or bind the cortical cytoskeletal protein CASK. The former could facilitate formation of specific protein complexes, whereas the latter, together with protein 4.1 , would place these complexes at the plasma membrane in proximity to transsynaptic adhesion molecules, such as $\mathrm{N}$-cadherin and integrins. Impor-
A

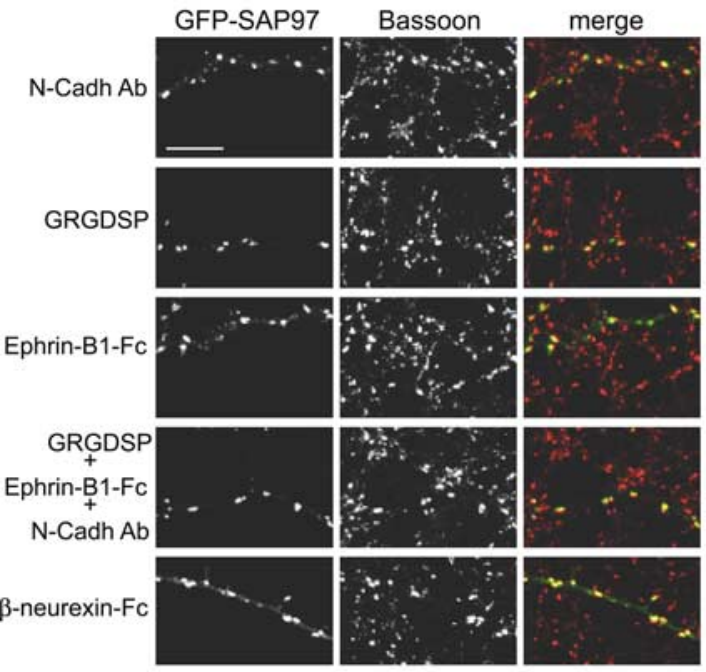

B

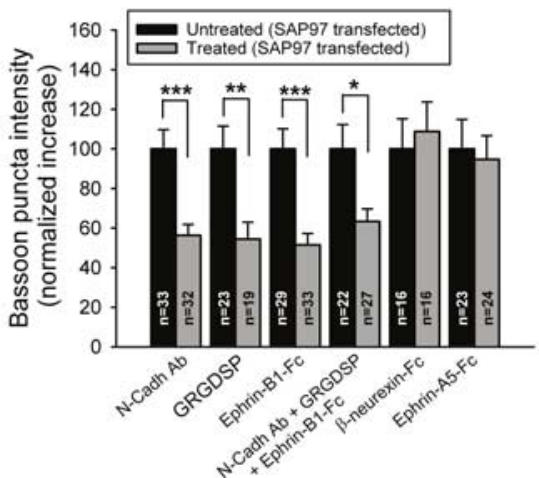

C

C1

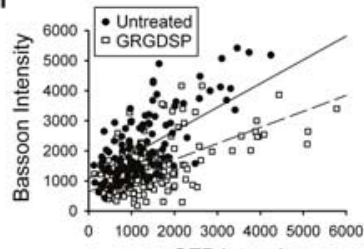

C2

GFP intensity

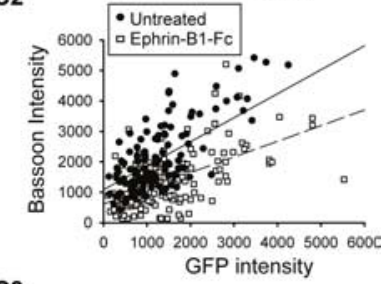

C3

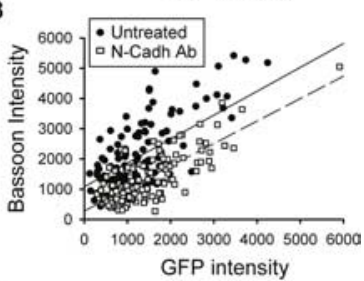

D

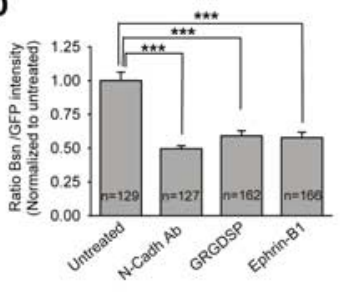

Figure 9. Inhibition of transsynaptic interactions of $\mathrm{N}$-cadherins, integrins, or EphB receptor/ephrinB1 decreases the presynaptic effects of postsynaptic SAP97. $A$, Representative images of neurons transfected with GFP-SAP97 and treated for $3 \mathrm{~d}$ with different reagents, as indicated on the left. Each row of images shows GFP fluorescence (left), staining for Bassoon (middle), and merged images (right). Scale bar, $10 \mu \mathrm{m}$. B, Quantification of the average Bassoon puncta intensity in neurons expressing intensity that colocalize with GFP-SAP97 from control, untreated cultures. The gray bars show the average increase of Bassoon puncta intensity that colocalize with GFP-SAP97 in treated cultures. The increase was normalized to that observed in sister contro unclustered $\beta$-neurexin-Fc or Ephrin-A5-Fc failed to prevent the SAP97-mediated increase in Bassoon staining compared with untreated sister cultures. Treatment with a function blocking antibody for $\mathrm{N}$-cadherin ( $\mathrm{N}$-Cadh $\mathrm{Ab}$ ), a peptide that inhibits integrin resynaptic effects of SAP97 (mean \pm SEM; $n=$ number of cells; ${ }^{* * *} p<0.0005,{ }^{* *} p<0.005,{ }^{*} p<0.05$, two-tailed $t$ test). $C$

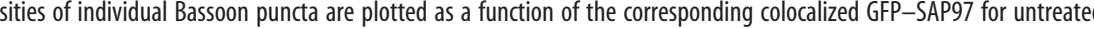
of Bassoon (Bsn)/GFP intensity ratio for individual puncta expressing GFP-SAP97 in untreated and treated cultures. Comparisons were made between transfected neurons from sister cultures. Data were normalized to the Bsn/GFP-SAP97 ratio in untreated cultures (mean $\pm \mathrm{SEM} ; n=$ number of puncta; same data as in $C$ ). ${ }^{* * *} p<0.0005$, two-tailed $t$ test.

tantly, SAP97, cadherins, and integrins are all associated with the actin/spectrin cortical cytoskeleton. In fact, in non-neuronal cell systems, SAP97 is recruited to sites of cell-cell adhesion in a cadherin dependent manner (Reuver and Garner, 1998) and promotes cell-cell adhesion (Thomas et al., 1997; Firestein and Rongo, 2001).

It is unclear at this point how the SAP97 PDZ domains, in particular PDZ1 and PDZ2, contribute to the transsynaptic effects of postsynaptic SAP97. One possibility is that the critical transsynaptic signaling is mediated by the recruitment of proteins that bind to the PDZ domains such as neuroligin (Irie et al., 
1997), the ras GDP/GTP exchange protein SynGAP (Kim et al., 1998), NR2A/2B subunits of the NMDAR (Gardoni et al., 2003), or the Rac guanine nucleotide exchange factor kalirin-7 (Penzes et al., 2001). Of these, neuroligin seems the least likely to be involved, because its binding pocket in the third PDZ3 domain was not required for SAP97 to exert its presynaptic effects. Moreover, addition of $\beta$-neurexin-Fc to the cultures, a manipulation that disrupts retrograde signaling between neuroligins and their presynaptic partner $\beta$-neurexin (Scheiffele et al., 2000), had no effect on the presynaptic actions of SAP97. With regard to other PDZ domain-binding proteins, both NMDARs and kalirin activity are regulated by EphB receptors (Dalva et al., 2000; Takasu et al., 2002; Penzes et al., 2003), which our results suggest contribute to the presynaptic effects of postsynaptic SAP97.

A final region of SAP97 found to be important for transducing its presynaptic effects is the GK domain, which binds members of the GK associated protein/SAP90/PSD-95-associated protein (GKAP/SAPAP) family (Kim et al., 1997; Takeuchi et al., 1997) and SPAR/SPAL, an actin binding protein with RapGAP activity (Pak et al., 2001; Roy et al., 2002). That these interactions may contribute to the presynaptic effects of SAP97 are suggested by three sets of findings. First, postsynaptic levels of SPAR/SPAL were elevated by overexpression of SAP97 as were levels of ProSAP/Shank family members (proSAP2 and Shank1a), which are directed to their postsynaptic localization via their interactions with GKAP/SAPAP (Boeckers et al., 1999; Naisbitt et al., 1999). Second, postsynaptic overexpression of Shankla, ProSAP2/ Shank3, or SPAR/SPAL not only promote the maturation of dendritic spines but also cause additional changes consistent with presynaptic growth (Pak et al., 2001; Sala et al., 2001). Third, SPAR/SPAL can regulate the establishment and maintenance of cell adhesion (Tsukamoto et al., 1999) through the activation of integrins (Caron et al., 2000; Reedquist et al., 2000). Thus, raising the levels of SPAR/SPAL postsynaptically might be expected to increase integrin mediated adhesion, which our results (Fig. 9), as well as previous results (Chavis and Westbrook, 2001), suggest could function as a transsynaptic signal for presynaptic maturation and growth.

Our results do not allow us to conclude which of the postsynaptic binding partners of SAP97 are critically involved in mediating its presynaptic effects. However, experiments using inhibitors of transsynaptic protein interactions provided information about which sets of molecules are important for the postsynaptic to presynaptic retrograde signaling that is responsible for the enlargement of presynaptic terminals caused by SAP97. Interfering with the homophilic interactions between $\mathrm{N}$-cadherins and integrins reduced the presynaptic effects of postsynaptic SAP97 as did interfering with Ephrin B1/EphB receptor interactions. Surprisingly, combining these three treatments did not have a greater affect than either treatment alone. Thus, additional transsynaptic interactions may be involved. These results suggest that the maturation and growth of excitatory synapses involves the cooperative interplay of several transsynaptic protein interactions that are partially redundant. Such a mechanism may have evolved to ensure synapse stability such that mutations in single transsynaptic pathways cannot profoundly interfere with synaptic function and thereby survival.

In conclusion, we have demonstrated that postsynaptic SAP97 can influence presynaptic structure and function, as evidenced by an increase in the levels of three different classes of presynaptic proteins and enhanced FM4-64 uptake. SAP97 appears to induce these changes through a complex set of events that capitalizes on its multidomain structure to promote the assembly of specific protein complexes, a concept supported by the differences observed between SAP97, SAP102, and PSD-95. Mechanistically, SAP97 appears to promote presynaptic changes by engaging a combination of transsynaptic signaling molecules such as $\mathrm{N}$-cadherin, integrin, and ephrinB/EphB. This may be attributable, in part, to the ability of SAP97 to increase the synaptic levels of several other postsynaptic proteins such as Shank1a, ProSAP2, and SPAR/SPAL. Together, these data suggest that the structural and functional presynaptic and postsynaptic changes that occur during development and in response to activity likely involve a complex yet coordinated interplay of multiple different transsynaptic protein interactions. Further dissecting these mechanisms is a challenging endeavor that should be facilitated by the types of molecular manipulations performed here.

\section{References}

Belichenko PV, Masliah E, Kleschevnikov AM, Villar AJ, Epstein CJ, Salehi A, Mobley WC (2004) Synaptic structural abnormalities in the Ts65Dn mouse model of Down syndrome. J Comp Neurol 480:281-298.

Betz WJ, Bewick GS (1992) Optical analysis of synaptic vesicle recycling at the frog neuromuscular junction. Science 255:200-203.

Boeckers TM, Winter C, Smalla KH, Kreutz MR, Bockmann J, Seidenbecher C, Garner CC, Gundelfinger ED (1999) Proline-rich synapse-associated proteins ProSAP1 and ProSAP2 interact with synaptic proteins of the SAPAP/GKAP family. Biochem Biophys Res Commun 264:247-252.

Boeckers TM, Bockmann J, Kreutz MR, Gundelfinger ED (2002) ProSAP/ Shank proteins-a family of higher order organizing molecules of the postsynaptic density with an emerging role in human neurological disease. J Neurochem 81:903-910.

Cai C, Coleman SK, Niemi K, Keinanen K (2002) Selective binding of synapse-associated protein 97 to GluR-A alpha-amino-5-hydroxy-3methyl-4-isoxazole propionate receptor subunit is determined by a novel sequence motif. J Biol Chem 277:31484-31490.

Caron E, Self AJ, Hall A (2000) The GTPase Rap1 controls functional activation of macrophage integrin alphaMbeta2 by LPS and other inflammatory mediators. Curr Biol 10:974-978.

Chavis P, Westbrook G (2001) Integrins mediate functional pre- and postsynaptic maturation at a hippocampal synapse. Nature 411:317-321.

Chen L, Chetkovich DM, Petralia RS, Sweeney NT, Kawasaki Y, Wenthold RJ, Bredt DS, Nicoll RA (2000) Stargazing regulates synaptic targeting of AMPA receptors by two distinct mechanisms. Nature 408:936-943.

Contractor A, Rogers C, Maron C, Henkemeyer M, Swanson GT, Heinemann SF (2002) Trans-synaptic Eph receptor-ephrin signaling in hippocampal mossy fiber LTP. Science 296:1864-1869.

Dalva MB, Takasu MA, Lin MZ, Shamah SM, Hu L, Gale NW, Greenberg ME (2000) EphB receptors interact with NMDA receptors and regulate excitatory synapse formation. Cell 103:945-956.

Doyle DA, Lee A, Lewis J, Kim E, Sheng M, MacKinnon R (1996) Crystal structures of a complexed and peptide-free membrane protein-binding domain: molecular basis of peptide recognition by PDZ. Cell 85:1067-1076.

El-Husseini AE, Schnell E, Chetkovich DM, Nicoll RA, Bredt DS (2000) PSD-95 involvement in maturation of excitatory synapses. Science 290:1364-1368.

Engert F, Bonhoeffer T (1999) Dendritic spine changes associated with hippocampal long-term synaptic plasticity. Nature 399:66-70.

Firestein BL, Rongo C (2001) DLG-1 is a MAGUK similar to SAP97 and is required for adherens junction formation. Mol Biol Cell 12:3465-3475.

Gardoni F, Mauceri D, Fiorentini C, Bellone C, Missale C, Cattabeni F, Di Luca M (2003) CaMKII-dependent phosphorylation regulates SAP97/ NR2A interaction. J Biol Chem 278:44745-44752.

Garner CC, Zhai RG, Gundelfinger ED, Ziv NE (2002) Molecular mechanisms of CNS synaptogenesis. Trends Neurosci 25:243-251.

Hanada T, Takeuchi A, Sondarva G, Chishti AH (2003) Protein 4.1mediated membrane targeting of human discs large in epithelial cells. J Biol Chem 278:34445-34450.

Harris BZ, Lim WA (2001) Mechanism and role of PDZ domains in signaling complex assembly. J Cell Sci 114:3219-3231.

Irie M, Hata Y, Takeuchi M, Ichtchenko K, Toyoda A, Hirao K, Takai Y, 
Rosahl TW, Sudhof TC (1997) Binding of neuroligins to PSD-95. Science 277:1511-1515.

Kim E, Sheng M (2004) PDZ domain proteins of synapses. Nat Rev Neurosci 5:771-781.

Kim E, Naisbitt S, Hsueh YP, Rao A, Rothschild A, Craig AM, Sheng M (1997) GKAP, a novel synaptic protein that interacts with the guanylate kinase-like domain of the PSD-95/SAP90 family of channel clustering molecules. J Cell Biol 136:669-678.

Kim JH, Liao D, Lau LF, Huganir RL (1998) SynGAP: a synaptic RasGAP that associates with the PSD-95/SAP90 protein family. Neuron 20:683-691.

Lawrenson ID, Wimmer-Kleikamp SH, Lock P, Schoenwaelder SM, Down M, Boyd AW, Alewood PF, Lackmann M (2002) Ephrin-A5 induces rounding, blebbing and de-adhesion of EphA3-expressing 293T and melanoma cells by CrkII and Rho-mediated signalling. J Cell Sci 115:1059-1072.

Lee S, Fan S, Makarova O, Straight S, Margolis B (2002) A novel and conserved protein-protein interaction domain of mammalian Lin-2/CASK binds and recruits SAP97 to the lateral surface of epithelia. Mol Cell Biol 22:1778-1791.

Leonard AS, Davare MA, Horne MC, Garner CC, Hell JW (1998) SAP97 is associated with the alpha-amino-3-hydroxy-5-methylisoxazole-4propionic acid receptor GluR1 subunit. J Biol Chem 273:19518-19524.

Li Z, Sheng M (2003) Some assembly required: the development of neuronal synapses. Nat Rev Mol Cell Biol 4:833-841.

Lim IA, Hall DD, Hell JW (2002) Selectivity and promiscuity of the first and second PDZ domains of PSD-95 and synapse-associated protein 102. J Biol Chem 277:21697-21711.

Lisman JE, Harris KM (1993) Quantal analysis and synaptic anatomy-integrating two views of hippocampal plasticity. Trends Neurosci 16:141-147.

Long JF, Tochio H, Wang P, Fan JS, Sala C, Niethammer M, Sheng M, Zhang M (2003) Supramodular structure and synergistic target binding of the N-terminal tandem PDZ domains of PSD-95. J Mol Biol 327:203-214.

Lue RA, Marfatia SM, Branton D, Chishti AH (1994) Cloning and characterization of hdlg: the human homologue of the Drosophila discs large tumor suppressor binds to protein 4.1. Proc Natl Acad Sci USA 91:9818-9822.

Marfatia SM, Byron O, Campbell G, Liu SC, Chishti AH (2000) Human homologue of the Drosophila discs large tumor suppressor protein forms an oligomer in solution. Identification of the self-association site. J Biol Chem 275:13759-13770.

Matsuzaki M, Honkura N, Ellis-Davies GC, Kasai H (2004) Structural basis of long-term potentiation in single dendritic spines. Nature 429:761-766.

McLaughlin M, Hale R, Ellston D, Gaudet S, Lue RA, Viel A (2002) The distribution and function of alternatively spliced insertions in hDlg. J Biol Chem 277:6406-6412.

Montgomery JM, Zamorano PL, Garner CC (2004) MAGUKs in synapse assembly and function: an emerging view. Cell Mol Life Sci 61:911-929.

Muller BM, Kistner U, Veh RW, Cases-Langhoff C, Becker B, Gundelfinger ED, Garner CC (1995) Molecular characterization and spatial distribution of SAP97, a novel presynaptic protein homologous to SAP90 and the Drosophila discs-large tumor suppressor protein. J Neurosci 15:2354-2366.

Nagerl UV, Eberhorn N, Cambridge SB, Bonhoeffer T (2004) Bidirectional activity-dependent morphological plasticity in hippocampal neurons. Neuron 44:759-767.

Naisbitt S, Kim E, Tu JC, Xiao B, Sala C, Valtschanoff J, Weinberg RJ, Worley PF, Sheng M (1999) Shank, a novel family of postsynaptic density proteins that binds to the NMDA receptor/PSD-95/GKAP complex and cortactin. Neuron 23:569-582.

Nakagawa T, Futai K, Lashuel HA, Lo I, Okamoto K, Walz T, Hayashi Y, Sheng M (2004) Quaternary structure, protein dynamics, and synaptic function of SAP97 controlled by L27 domain interactions. Neuron 44:453-467.

Niethammer M, Kim E, Sheng M (1996) Interaction between the C terminus of NMDA receptor subunits and multiple members of the PSD-95 family of membrane-associated guanylate kinases. J Neurosci 16:2157-2163.

Niethammer M, Valtschanoff JG, Kapoor TM, Allison DW, Weinberg TM, Craig AM, Sheng M (1998) CRIPT, a novel postsynaptic protein that binds to the third PDZ domain of PSD-95/SAP90. Neuron 20:693-707.
Pak DT, Yang S, Rudolph-Correia S, Kim E, Sheng M (2001) Regulation of dendritic spine morphology by SPAR, a PSD-95-associated RapGAP. Neuron 31:289-303.

Penzes P, Johnson RC, Sattler R, Zhang X, Huganir RL, Kambampati V, Mains RE, Eipper BA (2001) The neuronal Rho-GEF Kalirin-7 interacts with PDZ domain-containing proteins and regulates dendritic morphogenesis. Neuron 29:229-242.

Penzes P, Beeser A, Chernoff J, Schiller MR, Eipper BA, Mains RE, Huganir RL (2003) Rapid induction of dendritic spine morphogenesis by transsynaptic ephrinB-EphB receptor activation of the Rho-GEF kalirin. Neuron 37:263-274.

Piserchio A, Pellegrini M, Mehta S, Blackman SM, Garcia EP, Marshall J, Mierke DF (2002) The PDZ1 domain of SAP90. Characterization of structure and binding. J Biol Chem 277:6967-6973.

Reedquist KA, Ross E, Koop EA, Wolthuis RM, Zwartkruis FJ, van Kooyk Y, Salmon M, Buckley CD, Bos JL (2000) The small GTPase, Rap1, mediates CD31-induced integrin adhesion. J Cell Biol 148:1151-1158.

Reuver S, Garner C (1998) E-cadherin mediated cell adhesion recruits SAP97 into the cortical cytoskeleton. J Cell Sci 111:1071-1080.

Roy BC, Kohu K, Matsuura K, Yanai H, Akiyama T (2002) SPAL, a Rapspecific GTPase activating protein, is present in the NMDA receptorPSD-95 complex in the hippocampus. Genes Cells 7:607-617.

Rumbaugh G, Sia GM, Garner CC, Huganir RL (2003) Synapse-associated protein-97 isoform-specific regulation of surface AMPA receptors and synaptic function in cultured neurons. J Neurosci 23:4567-4576.

Ruoslahti E (1996) RGD and other recognition sequences for integrins. Annu Rev Cell Dev Biol 12:697-715.

Ryan TA, Reuter H, Wendland B, Schweizer FE, Tsien RW, Smith SJ (1993) The kinetics of synaptic vesicle recycling measured at single presynaptic boutons. Neuron 11:713-724.

Sabio G, Arthur JS, Kuma Y, Peggie M, Carr J, Murray-Tait V, Centeno F, Goedert M, Morrice NA, Cuenda A (2005) p38gamma regulates the localisation of SAP97 in the cytoskeleton by modulating its interaction with GKAP. EMBO J 24:1134-1145.

Sala C, Piech V, Wilson NR, Passafaro M, Liu G, Sheng M (2001) Regulation of dendritic spine morphology and synaptic function by Shank and Homer. Neuron 31:115-130.

Scheiffele P (2003) Cell-cell signaling during synapse formation in the CNS. Annu Rev Neurosci 26:485-508.

Scheiffele P, Fan J, Choih J, Fetter R, Serafini T (2000) Neuroligin expressed in nonneuronal cells triggers presynaptic development in contacting axons. Cell 101:657-669.

Schnell E, Sizemore M, Karimzadegan S, Chen L, Bredt DS, Nicoll RA (2002) Direct interactions between PSD-95 and stargazin control synaptic AMPA receptor number. Proc Natl Acad Sci USA 99:13902-13907.

Shapira M, Zhai RG, Dresbach T, Bresler T, Torres VI, Gundelfinger ED, Ziv NE, Garner CC (2003) Unitary assembly of presynaptic active zones from Piccolo-Bassoon transport vesicles. Neuron 38:237-252.

Sheng M, Kim E (2000) The Shank family of scaffold proteins. J Cell Sci 113:1851-1856.

Songyang Z, Fanning AS, Fu C, Xu J, Marfatia SM, Chishti AH, Crompton A, Chan AC, Anderson JM, Cantley LC (1997) Recognition of unique carboxyl-terminal motifs by distinct PDZ domains. Science 275:73-77.

Sudhof TC (2004) The synaptic vesicle cycle. Annu Rev Neurosci 27:509-547.

Takasu MA, Dalva MB, Zigmond RE, Greenberg ME (2002) Modulation of NMDA receptor-dependent calcium influx and gene expression through EphB receptors. Science 295:491-495.

Takeuchi M, Hata Y, Hirao K, Toyoda A, Irie M, Takai Y (1997) SAPAPs. A family of PSD-95/SAP90-associated proteins localized at postsynaptic density. J Biol Chem 272:11943-11951.

Tang L, Hung CP, Schuman EM (1998) A role for the cadherin family of cell adhesion molecules in hippocampal long-term potentiation. Neuron 20:1165-1175.

Thomas U, Phannavong B, Muller B, Garner CC, Gundelfinger ED (1997) Functional expression of rat synapse-associated proteins SAP97 and SAP102 in Drosophila dlg-1 mutants: effects on tumor suppression and synaptic bouton structure. Mech Dev 62:161-174.

tom Dieck S, Sanmarti-Vila L, Langnaese K, Richter K, Kindler S, Soyke A, Wex H, Smalla KH, Kampf U, Franzer JT, Stumm M, Garner CC, Gundelfinger ED (1998) Bassoon, a novel zinc-finger CAG/glutamine- 
repeat protein selectively localized at the active zone of presynaptic nerve terminals. J Cell Biol 142:499-509.

Tsukamoto N, Hattori M, Yang H, Bos JL, Minato N (1999) Rapl GTPaseactivating protein SPA-1 negatively regulates cell adhesion. J Biol Chem 274:18463-18469.

Volknandt W (1995) The synaptic vesicle and its targets. Neuroscience 64:277-300.

Wu H, Reuver SM, Kuhlendahl S, Chung WJ, Garner CC (1998) Subcellular targeting and cytoskeletal attachment of SAP97 to the epithelial lateral membrane. J Cell Sci 111:2365-2376.

Wu H, Reissner C, Kuhlendahl S, Coblentz B, Reuver S, Kindler S, Gundelfinger ED, Garner CC (2000) Intramolecular interactions regulate SAP97 binding to GKAP. EMBO J 19:5740-5751.

Wu H, Nash JE, Zamorano P, Garner CC (2002) Interaction of SAP97 with minus-end-directed actin motor myosin VI. Implications for AMPA receptor trafficking. J Biol Chem 277:30928-30934.

Yu X, Malenka RC (2003) Beta-catenin is critical for dendritic morphogenesis. Nat Neurosci 6:1169-1177.

Yuste R, Bonhoeffer T (2001) Morphological changes in dendritic spines associated with long-term synaptic plasticity. Annu Rev Neurosci 24:1071-1089.

Zhai R, Olias G, Chung WJ, Lester RA, tom Dieck S, Langnaese K, Kreutz MR, Kindler S, Gundelfinger ED, Garner CC (2000) Temporal appearance of the presynaptic cytomatrix protein bassoon during synaptogenesis. Mol Cell Neurosci 15:417-428.

Zhou Q, Homma KJ, Poo MM (2004) Shrinkage of dendritic spines associated with long-term depression of hippocampal synapses. Neuron 44: 749-757. 